\title{
Mycophenolic acid pharmacogenomics in kidney transplantation
}

\author{
Fabiana Dalla Vecchia Genvigir ${ }^{1}$, Alvaro Cerda ${ }^{2}$, Thiago Dominguez Crespo Hirata ${ }^{1}$, Mario Hiroyuki Hirata ${ }^{1}$, \\ Rosario Dominguez Crespo Hirata ${ }^{1}$ \\ 'Department of Clinical and Toxicological Analyses, School of Pharmaceutical Sciences, University of Sao Paulo, Sao Paulo \\ 05508-000, Brazil. \\ 2Department of Basic Sciences, Center of Excellence in Translational Medicine, CEMT-BIOREN, Universidad de La Frontera, \\ Temuco 4810296, Chile.
}

Correspondence to: Prof. Rosario Dominguez Crespo Hirata, Department of Clinical and Toxicological Analyses, School of Pharmaceutical Sciences, University of Sao Paulo, Av. Prof. Lineu Prestes 580, Sao Paulo 05508-000, Brazil.

E-mail: rosariohirata@usp.br

\begin{abstract}
How to cite this article: Genvigir FDV, Cerda A, Hirata TDC, Hirata MH, Hirata RDC. Mycophenolic acid pharmacogenomics in kidney transplantation. J Trans/ Genet Genom 2020;4:320-55. http://dx.doi.org/10.20517/jtgg.2020.37
\end{abstract}

Received: 12 Jun 2020 First Decision: 23 Jul 2020 Revised: 30 Jul 2020 Accepted: 17 Aug 2020 Available online: 28 Aug 2020

Academic Editor: Ramón Cacabelos Copy Editor: Cai-Hong Wang Production Editor: Jing Yu

\begin{abstract}
Mycophenolic acid (MPA) is a potent antiproliferative drug prescribed to prevent acute rejection in kidney transplantation. MPA reversibly inhibits the enzymes involved in the synthesis of guanosine nucleotides, thus preventing DNA replication of immune cells. Consequently, the repression of both cell and humoral immunity induces renal allograft tolerance. MPA is an effective and safe immunosuppressive drug, but some patients show variability in drug concentration, acute rejection, graft dysfunction, or MPA-related adverse events. Although the pharmacogenomics of immunosuppressive drugs has been widely investigated, MPA has been explored to a lesser extent. This review of MPA pharmacogenomic studies, included pharmacokinetics, adverse events, and main clinical outcomes of MPA treatment in kidney transplantation. Associations of variants in genes encoding MPA metabolizing enzymes, transporters, and targets with drug efficacy and safety are described. Most pharmacogenetic studies have focused on small sample sizes and few simultaneously analyzed genetic variants. Some studies reported significant associations of pharmacokinetics- and pharmacodynamics-related genes with MPA exposure, acute rejection, graft dysfunction, hematological events, and gastrointestinal complications. However, even large cohorts did not replicate the findings, possibly due to divergent study design, immunosuppressive scheme, follow-up time, and other factors. Finally, the heterogeneity of aspects between studies limit conclusions on pharmacogenetic biomarkers of MPA in kidney transplantation.
\end{abstract}

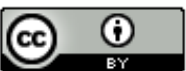

(C) The Author(s) 2020. Open Access This article is licensed under a Creative Commons Attribution 4.0 International License (https://creativecommons.org/licenses/by/4.0/), which permits unrestricted use, sharing, adaptation, distribution and reproduction in any medium or format, for any purpose, even commercially, as long as you give appropriate credit to the original author(s) and the source, provide a link to the Creative Commons license, and indicate if changes were made. 
Keywords: Immunosuppressive therapy, mycophenolic acid, kidney transplantation, pharmacogenomics, pharmacodynamics, pharmacokinetics

\section{INTRODUCTION}

Mycophenolic acid (MPA) is a potent antiproliferative drug broadly prescribed to prevent acute rejection in kidney transplantation. MPA is a reversible inhibitor of inosine- $5^{\prime}$-monophosphate dehydrogenase (IMPDH), an important enzyme involved in the de novo synthesis of guanosine nucleotides, which are essential for the proliferation of $\mathrm{T}$ and $\mathrm{B}$ cells $^{[1,2]}$. Consequently, guanosine nucleotide depletion by MPA prevents DNA replication, leads to repression of both cell-and humoral-mediated immunity and induces tolerance to allograft in kidney transplantation ${ }^{[1,3,4]}$.

IMPDH activity results from the expression of two isoforms, IMPDH type I and type II, which are encoded by $I M P D H_{1}$ and $I M P D H_{2}$, respectively ${ }^{[2]}$. Both genes are constitutively expressed in most tissues, though activated $\mathrm{T}$ and $\mathrm{B}$ lymphocytes have a higher expression of $I M P D H 2^{[1]}$. Also, IMPDH type II has a higher affinity for MPA compared to IMPDH type I, which makes it a selective and potent antiproliferative drug for $\mathrm{T}$ and $\mathrm{B}$ cells $^{[1,3]}$ [Figure 1].

MPA is available either as an ester prodrug or as a sodium salt, which are equivalent in terms of therapeutic effect and MPA exposure ${ }^{[5]}$. The prodrug 2-morpholinoethyl ester, named mycophenolate mofetil (MMF), is converted to the active metabolite MPA by carboxylesterases 1 and 2 (CES1 and CES2) ${ }^{[6]}$.

MPA is considered a safe drug even though some adverse events may occur, such as gastrointestinal complications, myelotoxicity, susceptibility to infections and neoplasms ${ }^{[1,3]}$. Designed to reduce gastrointestinal adverse events, the enteric-coated mycophenolate sodium salt (EC-MPS) has a delayed release formulation that delivers mycophenolate in the small intestine ${ }^{[7]}$. Due to enteric coating, EC-MPS causes a slower absorption than MMF, and more variable time for MPA to reach maximal concentration ${ }^{[8]}$.

MPA is extensively converted (about 90\%) to the inactive 7-O-glucuronide (MPAG), by UDPglucuronosyltransferases (UGTs), mainly in the liver but also in intestine and to a minor extent in the kidney $^{[9]}$. This process is mediated in the liver and kidney by UGT1A9, whereas UGT1A8 plays a key role in the intestine with minor contribution from the other UGTs ${ }^{[9]}$ [Figure 1]. Since MPA and MPAG are bound to serum albumin, an accumulation of MPAG may compete with free MPA for albumin and lead to increased free MPA concentration in plasma ${ }^{[9,10]}$.

UGT2B7, with minor contribution of UGT1A8, mediates the biotransformation of MPA to its acylglucuronide form (AcMPAG) [Figure 1]. This metabolite can inhibit the activity of IMPDH and is therefore pharmacologically active ${ }^{[11]}$. The cytochrome P450 (CYP) family also plays a role in MPA biotransformation. The 6-O-desmethyl-MPA (DM-MPA) is a phase I metabolite of MPA produced in the liver by the activity of CYP3A4, CYP3A5, and to a lesser extent by $\mathrm{CYP} 2 \mathrm{C}^{[12]}$.

MPAG and AcMPAG, but not MPA, are excreted in the bile. The incorporation of these metabolites into hepatocytes is mediated by organic anion transport polypeptides (OATPs) ${ }^{[13,14]}$, which are membrane influx transporters encoded by genes of the SLCO family. From the hepatocyte, MPAG and AcMPAG are excreted in the bile via ATP-binding cassette subfamily $\mathrm{C}$ member 2 (ABCC2), also named multidrug resistanceassociated protein $2(\mathrm{MRP} 2)^{[15,16]}$. ABC transporters are ATP-dependent drug efflux pumps ${ }^{[17]}$. Another important member is ABCB1 (also known as P-glycoprotein or multidrug resistance protein 1-MDR1), which seems to play a role in MPA disposition ${ }^{[18]}$. 
Enterohepatic circulation

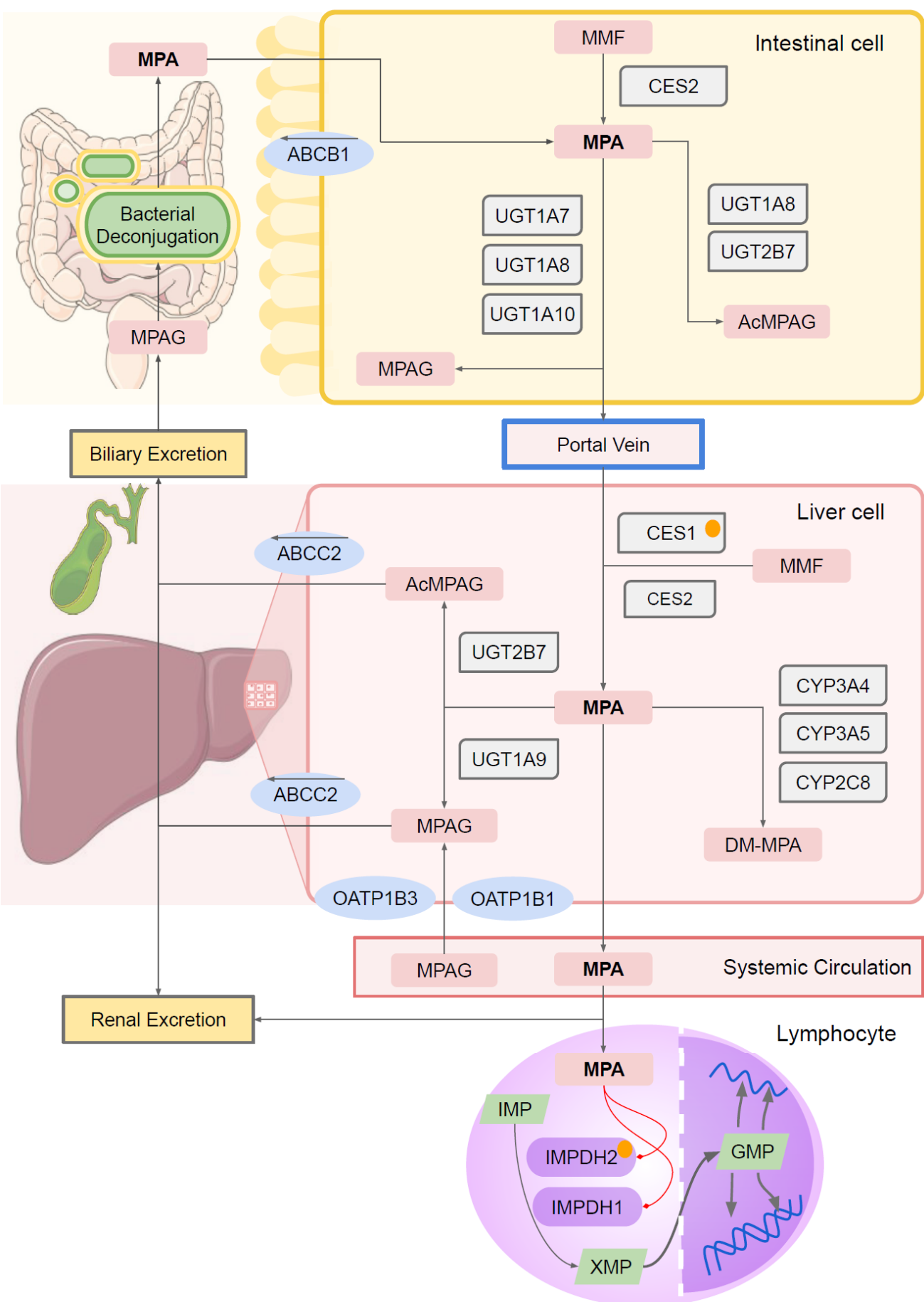

Figure 1. Schematic representation of MPA pharmacokinetics pathways and mechanisms of action. AcMPAG: acyl-MPA glucuronide; DM-MPA: 6-O-desmethyl MPA; GMP: guanosine 5'-monophosphate; IMP: inosine 5'-monophosphate; MMF: mycophenolate mofetil; MPA: mycophenolic acid; MPAG: MPA-7-O-glucuronide; XMP: xanthosine 5'-monophosphate

MPAG excreted in the bile undergoes extensive enterohepatic recirculation, being hydrolyzed to MPA in the small intestine and reabsorbed [Figure 1]. The enterohepatic recirculation contributes 10\%-60\% of the total MPA exposure and causes a secondary peak 6-12 $\mathrm{h}$ after oral MPA administration ${ }^{[1]}$. MPA is also eliminated in the urine as MPA in negligible amounts, and mostly as MPAG, possibly mediated by $\mathrm{ABCC} 2^{[19]}$. 
The more common MPA-related adverse events are diarrhea and other gastrointestinal complications. MMF can also cause myelotoxicity, especially leukopenia and anemia. Susceptibility to viral infections, such as cytomegalovirus (CMV) and BK polyomavirus (BKV), also increases in patients taking an MMFcontaining triple therapy regimen. Patients with low tolerance to MPA may require dose reduction, temporary interruption, or permanent discontinuation of the MMF treatment ${ }^{[1]}$.

The contribution of pharmacogenomics in the response to immunosuppressive drugs has been widely investigated. Several clinical studies have reported the influence of gene polymorphisms on the efficacy and safety of MPA, suggesting their potential contribution to transplant patient management ${ }^{[1-3]}$.

This review explores the pharmacogenomic studies that investigated polymorphisms in genes involved in the pharmacokinetics and pharmacodynamics of MPA in kidney transplantation and the main clinical outcomes and adverse events.

\section{GENES RELATED TO MPA PHARMACOKINETICS}

The pharmacogenomic studies involving genes related to MPA-metabolizing enzymes and transporters are summarized in Tables 1 and 2, respectively.

\section{CES1 and CES2}

CES1 and CES2 play crucial roles in the hydrolysis of the MMF prodrug to MPA. CES1 is highly expressed in the liver, while showing extremely low levels in the intestine. On the other hand, CES2 is mainly expressed in the intestine, and also observed in liver tissue ${ }^{[20]}$.

Fujiyama et al ${ }^{[21]}$ evaluated the influence of CES2 variants in $5^{\prime} \mathrm{UTR}(-1548 \mathrm{~A}>\mathrm{G}, \mathrm{rs} 3890213)$ and intronic regions (4595A>G, rs2303218 and $8721 \mathrm{C}>\mathrm{T}$, rs2241409) on MPA pharmacokinetics. They found no association of these variants with MPA plasma concentration-time area under the curve, partial $\left(\mathrm{AUC}_{0-6}\right)$ and total $\left(\mathrm{AUC}_{0-12}\right)$, maximum plasma concentration $(\mathrm{Cmax})$ and time required to reach the peak (Tmax) in 80 Japanese kidney recipients at 28 days after transplantation. These variants were also not associated with allograft rejection (AR) or diarrhea ${ }^{[21]}$.

In a retrospective study with Brazilian patients a long time after transplantation ( $7.00 \pm 5.71$ years), CES2 8721 TT genotype was associated with increased risk of AR (OR: 7.2; 95\%CI: 1.1-46, $P=0.038$ ), whereas the CES1 356C>T (rs62028647) variant was not associated with $\mathrm{AR}^{[22]}$.

\section{CYP3A4, CYP3A5 and CYP2C8}

The CYP enzymes CYP3A4, CYP3A5 and possibly CYP2C8 can catalyze the generation of the phase 1 metabolite DM-MPA, evident in human liver samples ${ }^{[12]}$, suggesting that variants in these genes may be involved in the clinical outcomes of MPA treatment.

Studies with certain variants in CYP2C8 (rs11572080 and rs10509681), CYP3A4 (rs2740574 and rs35599367) and CYP3A5 (rs776746) found no association with MPA trough concentration/dose ratio (C/D) and $\mathrm{AUC}_{0-12}$ in kidney transplantation ${ }^{[2,24]}$.

Although the CYP3A4 and CYP3A5 variants did not influence the clinical outcomes of MPA-based therapy ${ }^{[2-27]}$, the results were less consistent regarding $C Y P_{2} C 8$ variants.

CYP2C8 $\operatorname{rs} 11572076(\mathrm{G}>\mathrm{A}$ ) variant was associated with increased risk of anemia (OR: 3.2, 95\%CI: 1.7-6.2, $P<0.001)$ in a study that evaluated 2,724 variants in 978 adult kidney or simultaneous kidney-pancreas recipients ${ }^{[28]}$. The authors also found no association between this variant and leukopenia, a finding later 
Table 1. Genes related to mycophenolic acid metabolism in kidney transplantation

\begin{tabular}{|c|c|c|c|c|c|c|c|c|}
\hline Gene & Variant & Allele frequency & Study design & Population & $\begin{array}{c}\text { Immunosuppressive } \\
\text { regimen }\end{array}$ & Pharmacokinetics & Clinical outcomes & Ref. \\
\hline CES1 & rs62028647 (356C>T) & 356T: 46\% & $\begin{array}{l}\text { Retrospective (Case- } \\
\text { control) }\end{array}$ & $\begin{array}{l}145 \text { adult patients } \\
\text { (Brazil) }\end{array}$ & $\begin{array}{l}\text { MMF } \\
\text { Cyclosporine Tacrolimus } \\
\text { Sirolimus } \\
\text { Corticosteroids }\end{array}$ & - & $\begin{array}{l}\text { No association of CES1 } \\
356 C>T \text { variant with AR }\end{array}$ & [22] \\
\hline \multirow[t]{2}{*}{ CES2 } & $\begin{array}{l}r s 3890213 \\
(-1548 A>G) \\
r s 2303218(4595 A>G)\end{array}$ & - & Prospective & 80 adult patients (Japan) & $\begin{array}{l}\text { MMF } \\
\text { Tacrolimus } \\
\text { Corticosteroids } \\
\text { At day } 28\end{array}$ & $\begin{array}{l}\text { No association of } \\
\text { CES2 }-1548 A>G \text {, } \\
4595 A>G \text { and } 8721 C>T \\
\text { variants with MPA } \\
\text { pharmacokinetics }\end{array}$ & $\begin{array}{l}\text { No association of } C E S 2 \\
-1548 A>G, 4595 A>G \text { and } \\
8721 C>T \text { variants with } A R \\
\text { or diarrhea }\end{array}$ & [21] \\
\hline & rs2241409 (8721C>T) & $8721 \mathrm{~T}: 22 \%$ & $\begin{array}{l}\text { Retrospective (Case- } \\
\text { control) }\end{array}$ & $\begin{array}{l}145 \text { adult patients } \\
\text { (Brazil) }\end{array}$ & $\begin{array}{l}\text { MMF } \\
\text { Cyclosporine Tacrolimus } \\
\text { Sirolimus } \\
\text { Corticosteroids }\end{array}$ & - & $\begin{array}{l}\text { CES2 } 8721 T T \text { genotype was } \\
\text { associated with increased } \\
\text { risk of AR }\end{array}$ & {$[22]$} \\
\hline \multirow[t]{4}{*}{ CYP2C8 } & rs11572076 G>A & - & $\begin{array}{l}\text { Retrospective (Case- } \\
\text { Control), multicenter }\end{array}$ & $\begin{array}{l}284 \text { pediatric and young } \\
\text { adult patients (USA) }\end{array}$ & $\begin{array}{l}\text { MMF } \\
\text { Cyclosporine Tacrolimus } \\
\text { Sirolimus } \\
\text { Corticosteroids } \\
\text { One-year follow-up }\end{array}$ & - & $\begin{array}{l}\text { No association of } C Y P 2 C 8 \\
\text { rs } 11572076 \text { variant with } \\
\text { leukopenia }\end{array}$ & [27] \\
\hline & & rs11572076A: 0.5\% & $\begin{array}{l}\text { Prospective, multicenter } \\
\text { (Dominos study) }\end{array}$ & $\begin{array}{l}189 \text { adult patients } \\
\text { (France) }\end{array}$ & $\begin{array}{l}\text { EC-MPS } \\
\text { Cyclosporine } \\
\text { Corticosteroids } \\
\text { Six-month follow-up }\end{array}$ & - & $\begin{array}{l}\text { CYP2C8 rs11572076GG } \\
\text { genotype was associated } \\
\text { with reduced risk of } \\
\text { leukopenia. } \\
\text { No association with AR, } \\
\text { diarrhea, or anemia }\end{array}$ & [29] \\
\hline & $\begin{array}{l}\text { CYP2C8*3 } \\
{[\text { rs11572080 (c. } 416 G>A)} \\
+ \text { rs10509681 } \\
(c .1196 A>G)]\end{array}$ & CYP2C8*3: $9 \%$ & Prospective, multicenter & $\begin{array}{l}148 \text { adult patients } \\
\text { (Brazil) }\end{array}$ & $\begin{array}{l}\text { EC-MPS } \\
\text { Tacrolimus } \\
\text { Corticosteroids } \\
\text { Three-month follow-up }\end{array}$ & - & $\begin{array}{l}\text { CYP2C8*3 variant was } \\
\text { associated with higher } \\
\text { eGFR in CYP3A5-non- } \\
\text { expressing patients. } \\
\text { No association of } \\
\text { CYP2C } * 3 \text { variant with AR, } \\
\text { DGF or adverse events }\end{array}$ & [30] \\
\hline & & $\begin{array}{l}\text { c.416A: } 9 \% \\
\text { c.1196G:10\% }\end{array}$ & Prospective & 97 adult patients (Brazil) & $\begin{array}{l}\text { EC-MPS } \\
\text { Tacrolimus } \\
\text { Corticosteroid } \\
\text { One-year follow-up }\end{array}$ & $\begin{array}{l}\text { No association of } \\
\text { CYP2C } 8^{\star 3} \text { variant with } \\
\text { MPA C/D }\end{array}$ & $\begin{array}{l}\text { CYP2C } 8^{*} 3 \text { variant was } \\
\text { associated with higher } \\
\text { eGFR at week } 2 \text { and } \\
\text { month } 1 \text {. No association of } \\
\text { CYP2C } 8^{\star} 3 \text { variant with AR }\end{array}$ & [24] \\
\hline
\end{tabular}




\begin{tabular}{|c|c|c|c|c|c|c|c|c|}
\hline Gene & Variant & Allele frequency & Study design & Population & $\begin{array}{c}\text { Immunosuppressive } \\
\text { regimen }\end{array}$ & Pharmacokinetics & Clinical outcomes & Ref. \\
\hline \multirow[t]{4}{*}{$\overline{C Y P 3 A A 4}$} & $\begin{array}{l}\text { rs } 2740574\left(C Y P 3 A 4^{*} 7 B\right. \\
-392 A>G)\end{array}$ & $-392 \mathrm{G}: 5 \%$ & $\begin{array}{l}\text { Prospective, multicenter } \\
\text { (CAESAR study) }\end{array}$ & 237 adult patients & $\begin{array}{l}\text { MMF } \\
\text { Cyclosporine } \\
\text { Corticosteroids } \\
\text { One-year follow-up }\end{array}$ & - & $\begin{array}{l}\text { No association of } C Y P 3 A 4 \\
-392 A>G \text { variant with } A R\end{array}$ & [25] \\
\hline & & CYP3A4* $1 B(\mathrm{G}): 21 \%$ & Prospective & 97 adult patients (Brazil) & $\begin{array}{l}\text { EC-MPS } \\
\text { Tacrolimus } \\
\text { Corticosteroids } \\
\text { One-year follow-up }\end{array}$ & $\begin{array}{l}\text { No association of } \\
C Y P 3 A 4^{\star} T B \text { variant with } \\
\text { MPA C/D }\end{array}$ & $\begin{array}{l}\text { No association of } \\
\text { CYP } 3 A 4^{\star} 1 B \text { variant with } \\
\text { DGF or AR }\end{array}$ & [24] \\
\hline & $\begin{array}{l}\text { rs } 35599367 \\
\left(C Y P 3 A 4^{\star} 22\right)\end{array}$ & - & $\begin{array}{l}\text { Retrospective (Case- } \\
\text { Control), multicenter }\end{array}$ & $\begin{array}{l}284 \text { pediatric and young } \\
\text { adult patients (USA) }\end{array}$ & $\begin{array}{l}\text { MMF } \\
\text { Cyclosporine Tacrolimus } \\
\text { Sirolimus } \\
\text { Corticosteroids } \\
\text { One-year follow-up }\end{array}$ & - & $\begin{array}{l}\text { No association of } \\
\text { CYP } A A^{\star} 22 \text { variant with } \\
\text { leukopenia }\end{array}$ & [27] \\
\hline & & CYP $3 A 4^{\star} 22(\mathrm{~T}): 2 \%$ & Prospective & 97 adult patients (Brazil) & $\begin{array}{l}\text { EC-MPS } \\
\text { Tacrolimus } \\
\text { Corticosteroids } \\
\text { One-year follow-up }\end{array}$ & $\begin{array}{l}\text { No association of } \\
\text { CYPSA4*22 variant with } \\
\text { MPA C/D }\end{array}$ & $\begin{array}{l}\text { No association of } \\
\text { CYP } 3 A 4^{*} 22 \text { variant with } \\
\text { DGF or AR }\end{array}$ & [24] \\
\hline \multirow[t]{5}{*}{ CYPЗАS } & $\begin{array}{l}\text { rs } 776746 \\
(C Y P 3 A 5 * 3 \\
\text { g.6986A>G) }\end{array}$ & СУРЗА5*3(G):78\% & Prospective & 30 adult patients (Japan) & $\begin{array}{l}\text { MMF } \\
\text { Tacrolimus } \\
\text { Corticosteroids } \\
\text { Six-week follow-up }\end{array}$ & $\begin{array}{l}\text { No association of } \\
\text { CYP } 3 A 5^{\star} 3 \text { variant with } \\
\text { daytime and nighttime } \\
\text { MPA AUC }_{0-12}\end{array}$ & $\begin{array}{l}\text { No association of } \\
\text { CYP } 3 A 5^{\star} 3 \text { variant with } A R \\
\text { or gastrointestinal adverse } \\
\text { events }\end{array}$ & [23] \\
\hline & & CYP3A5`7(A): 9\% & $\begin{array}{l}\text { Prospective, multicenter } \\
\text { (CAESAR study) }\end{array}$ & 237 adult patients & $\begin{array}{l}\text { MMF } \\
\text { Cyclosporine } \\
\text { Corticosteroids } \\
\text { One-year follow-up }\end{array}$ & - & $\begin{array}{l}\text { No association of } \\
\text { CYP } 3 A 5^{\star} 3 \text { variant with } A R\end{array}$ & [25] \\
\hline & & СУРЗА5*3(G): $95 \%$ & Retrospective & $\begin{array}{l}207 \text { pediatric patients } \\
\text { (Poland) }\end{array}$ & $\begin{array}{l}\text { MMF or } \\
\text { Azathioprine } \\
\text { Cyclosporine Tacrolimus } \\
\text { Corticosteroids }\end{array}$ & - & $\begin{array}{l}\text { No association of } \\
\text { CYP } 3 A 5^{\star} 3 \text { variant with } \\
\text { MMF-related toxicity }\end{array}$ & [26] \\
\hline & & - & $\begin{array}{l}\text { Retrospective (Case- } \\
\text { Control), multicenter }\end{array}$ & $\begin{array}{l}284 \text { pediatric and young } \\
\text { adult patients (USA) }\end{array}$ & $\begin{array}{l}\text { MMF } \\
\text { Cyclosporine Tacrolimus } \\
\text { Sirolimus } \\
\text { Corticosteroids } \\
\text { One-year follow-up }\end{array}$ & - & $\begin{array}{l}\text { No association of } \\
\text { CYP } 3 A 5^{\star} 3 \text { variant with } \\
\text { leukopenia }\end{array}$ & [27] \\
\hline & & CYP3A5*7(A): $29 \%$ & Prospective & 97 adult patients (Brazil) & $\begin{array}{l}\text { EC-MPS } \\
\text { Tacrolimus } \\
\text { Corticosteroids } \\
\text { One-year follow-up }\end{array}$ & $\begin{array}{l}\text { No association of } \\
\text { CYP } 3 A 5^{*} 3 \text { variant with } \\
\text { MPA C/D }\end{array}$ & $\begin{array}{l}\text { No association of } \\
\text { CYP } 3 A 5^{\star} 3 \text { variant with } \\
\text { DGF or AR }\end{array}$ & [24] \\
\hline UGT1A1 & $\begin{array}{l}\text { rs3064744 } \\
\left(U G T 1 A 1^{\star 28}\right. \\
\text { A(TA)6TAA }> \\
\text { A(TA)7TAA })\end{array}$ & UGT1A1*28: 7\% & Prospective & $\begin{array}{l}30 \text { adult patients } \\
\text { (Japan) }\end{array}$ & $\begin{array}{l}\text { MMF } \\
\text { Tacrolimus } \\
\text { Corticosteroids } \\
\text { Six-week follow-up }\end{array}$ & $\begin{array}{l}\text { No association of } \\
U G T 1 A 1^{\star} 28 \text { variant with } \\
\text { daytime and nighttime } \\
\text { MPA AUC }_{0-12}\end{array}$ & $\begin{array}{l}\text { No association of } \\
U G T 1 A 7^{\star 2} 28 \text { variant with AR } \\
\text { or gastrointestinal adverse } \\
\text { events }\end{array}$ & [23] \\
\hline
\end{tabular}




\begin{tabular}{|c|c|c|c|c|c|c|c|c|}
\hline Gene & Variant & Allele frequency & Study design & Population & $\begin{array}{c}\text { Immunosuppressive } \\
\text { regimen }\end{array}$ & Pharmacokinetics & Clinical outcomes & Ref. \\
\hline \multirow[t]{4}{*}{ UGT1A7 } & $\begin{array}{l}r s 7586110 \\
(-57 T>G)\end{array}$ & - & $\begin{array}{l}\text { Retrospective (Case- } \\
\text { Control), multicenter }\end{array}$ & $\begin{array}{l}284 \text { pediatric and young } \\
\text { adult patients (USA) }\end{array}$ & $\begin{array}{l}\text { MMF } \\
\text { Cyclosporine Tacrolimus } \\
\text { Sirolimus } \\
\text { Corticosteroids } \\
\text { One-year follow-up }\end{array}$ & - & $\begin{array}{l}\text { No association of UGT1A7 } \\
-57 T>G \text { variant with } \\
\text { leukopenia }\end{array}$ & [27] \\
\hline & $\begin{array}{l}\text { rs11692021 (c.622T>C) } \\
U G T 1 A 7^{\star} 2[\mathrm{c} .387 T>\mathrm{G} \\
(\mathrm{rs} 17868323)+\end{array}$ & $\begin{array}{l}\text { UGT1A7*2: } 8 \% \\
\text { UGT1A7*3: } 28 \%\end{array}$ & Prospective & 80 adult patients (Japan) & $\begin{array}{l}\text { MMF } \\
\text { Tacrolimus } \\
\text { Corticosteroids } \\
\text { At day } 28\end{array}$ & $\begin{array}{l}\text { No association of } \\
\text { UGT1A }{ }^{\star} 2 \text { and } \\
\text { UGTIA } 7^{\star} 3 \text { variants with } \\
\text { MPA pharmacokinetics }\end{array}$ & - & [33] \\
\hline & $\begin{array}{l}\text { c.391C>A (rs17863778) } \\
+ \text { c.392G }>A \\
(r s 17868324)] \\
\text { UGT1A7*3 (c.387T>G + }\end{array}$ & c.622C: $37 \%$ & Retrospective & $\begin{array}{l}256 \text { adult patients } \\
\text { (France) }\end{array}$ & $\begin{array}{l}\text { MMF } \\
\text { Cyclosporine Tacrolimus } \\
\text { Sirolimus } \\
\text { Up to 115-month follow- } \\
\text { up }\end{array}$ & - & $\begin{array}{l}\text { No association of UGTIAT } \\
\text { c. } 622 C>\text { T variant with } \\
\text { gastrointestinal adverse } \\
\text { events }\end{array}$ & [34] \\
\hline & $\begin{array}{l}\text { c. } 391 C>A+c .392 G>A+ \\
\text { c. } 622 T>C)\end{array}$ & c.622C: $19 \%$ & Prospective & $\begin{array}{l}127 \text { adult patients } \\
\text { (China) }\end{array}$ & $\begin{array}{l}\text { MMF } \\
\text { Tacrolimus } \\
\text { Corticosteroids }\end{array}$ & $\begin{array}{l}\text { UGT1A7 c. } 622 C C \\
\text { genotype was associated } \\
\text { with higher } \\
\text { exposure to MPAG } \\
\left(\text { AUC }_{0-12}\right)\end{array}$ & $e^{-}$ & [35] \\
\hline \multirow[t]{5}{*}{ UGT1AB } & $\begin{array}{l}\text { rs17863762 }\left(U G T 1 A 8^{\star 3},\right. \\
\text { c. } 830 G>A) \\
\text { rs1042597 } \\
\left(U G T 1 A 8^{\star 2} 2, \text { c. } 518 C>G\right)\end{array}$ & - & Retrospective & $\begin{array}{l}74 \text { adult patients } \\
\text { (Brazil) }\end{array}$ & $\begin{array}{l}\text { MMF } \\
\text { Cyclosporine Tacrolimus } \\
\text { Sirolimus } \\
\text { Corticosteroids }\end{array}$ & - & $\begin{array}{l}\text { Treatment with MMF } 2 \mathrm{~g} \text { / } \\
\mathrm{d} \text { and UGT1A8 c.830AA } \\
\text { genotype were associated } \\
\text { with occurrence of } \\
\text { infections, but not diarrhea, } \\
\text { or blood disorders }\end{array}$ & {$[42]$} \\
\hline & & UGT1A8*2: $58 \%$ & Prospective & 72 adult patients (Japan) & $\begin{array}{l}\text { MMF } \\
\text { Tacrolimus } \\
\text { Corticosteroids } \\
\text { At day } 28\end{array}$ & $\begin{array}{l}\text { No association of } \\
\text { UGT1A } \mathcal{B}^{\star} 2 \text { variant with } \\
\text { daytime or nighttime } \\
\text { MPA pharmacokinetics }\end{array}$ & - & {$[36]$} \\
\hline & & c. 830 A: $4 \%$ & $\begin{array}{l}\text { Retrospective (Case- } \\
\text { Control) }\end{array}$ & $\begin{array}{l}38 \text { pediatric patients } \\
\text { (USA) }\end{array}$ & $\begin{array}{l}\text { MMF } \\
\text { Cyclosporine Tacrolimus } \\
\text { Corticosteroids }\end{array}$ & - & $\begin{array}{l}\text { No association of UGT1A8 } \\
\text { c. } 830 \mathrm{~A}>\mathrm{A} \text { variant with } \\
\text { adverse events (diarrhea or } \\
\text { leukopenia) }\end{array}$ & [43] \\
\hline & & $\begin{array}{l}\text { c. } 518 \mathrm{G}: 25 \% \\
\text { c. } 830 \mathrm{~A}: 2 \%\end{array}$ & $\begin{array}{l}\text { Prospective, multicenter } \\
\text { (FDCC Study) }\end{array}$ & 338 adult patients & $\begin{array}{l}\text { MMF } \\
\text { Cyclosporine Tacrolimus } \\
\text { Corticosteroids } \\
\text { One-year follow-up }\end{array}$ & $\begin{array}{l}\text { UGT1A } 8^{*} 2 /^{*} 2(\mathrm{GG}) \\
\text { genotype was associated } \\
\text { with higher MPA AUC } \\
\text { in cyclosporine-treated } \\
\text { patients }\end{array}$ & $\begin{array}{l}\text { No association of } U G T 1 A 8 \\
\text { c. } 518 \mathrm{C}>\mathrm{G} \text { and } \mathrm{c} .830 \mathrm{G}>\mathrm{A} \\
\text { variants with } \mathrm{AR}\end{array}$ & [39] \\
\hline & & c.518G: $26 \%$ & $\begin{array}{l}\text { Prospective (included } \\
\text { multicenter Apomygre } \\
\text { study) }\end{array}$ & $\begin{array}{l}185 \text { patients } \\
\text { (France) }\end{array}$ & $\begin{array}{l}\text { MMF } \\
\text { Cyclosporine } \\
\text { Tacrolimus } \\
\text { Sirolimus } \\
\text { Corticosteroids } \\
\text { At a point between 3-6 } \\
\text { months }\end{array}$ & $\begin{array}{l}\text { No association of } \\
\text { UGT1A } 8 \text { c. } 518 C>\mathrm{G} \\
\text { variant with MPA or } \\
\text { MPAG pharmacokinetics }\end{array}$ & - & [14] \\
\hline
\end{tabular}




\begin{tabular}{|c|c|c|c|c|c|c|c|c|}
\hline Gene & Variant & Allele frequency & Study design & Population & $\begin{array}{c}\text { Immunosuppressive } \\
\text { regimen }\end{array}$ & Pharmacokinetics & Clinical outcomes & Ref. \\
\hline & & $\begin{array}{l}\text { c. } 518 \mathrm{G}: 23 \% \\
\text { c. } 830 \mathrm{~A}: 2 \%\end{array}$ & Retrospective & $\begin{array}{l}256 \text { adult patients } \\
\text { (France) }\end{array}$ & $\begin{array}{l}\text { MMF } \\
\text { Cyclosporine Tacrolimus } \\
\text { Sirolimus } \\
\text { Up to 115-month follow- } \\
\text { up }\end{array}$ & - & $\begin{array}{l}\text { UGT1A8 c.518G allele was } \\
\text { associated with low risk of } \\
\text { diarrhea, on cyclosporine } \\
\text { treatment }\end{array}$ & [34] \\
\hline & & c. $830 \mathrm{~A}: 1 \%$ & Prospective, multicenter & $\begin{array}{l}89 \text { pediatric patients } \\
\text { (France) }\end{array}$ & $\begin{array}{l}\text { MMF } \\
\text { Cyclosporine Tacrolimus } \\
\text { Corticosteroids } \\
\text { Sixty-day follow-up }\end{array}$ & $\begin{array}{l}\text { No association of } \\
U G T 1 A 8 \text { c. } 830 G>A \\
\text { variant with MPA } \\
\text { pharmacokinetics }\end{array}$ & - & [40] \\
\hline & & c.518G: $43 \%$ & $\begin{array}{l}\text { Prospective, } \\
\text { Multicenter } \\
\text { (Case-control) }\end{array}$ & 37 adult patients (China) & $\begin{array}{l}\text { MMF } \\
\text { Cyclosporine } \\
\text { Corticosteroids }\end{array}$ & $\begin{array}{l}\text { No association of } \\
U G T 1 A 8 \text { c. } 518 C>G \\
\text { variant with } M P A \text { or } \\
\text { MPAG exposure }\left(A \cup C_{4-12}\right. \\
\text { or } A \cup C_{0-12} \text { ) }\end{array}$ & - & [37] \\
\hline & & c.518G: $32 \%$ & Prospective & $\begin{array}{l}109 \text { adult patients } \\
\text { (USA) }\end{array}$ & $\begin{array}{l}\text { MMF } \\
\text { EC-MPS } \\
\text { Cyclosporine Tacrolimus } \\
\text { Corticosteroids } \\
\text { Six-month follow-up }\end{array}$ & - & $\begin{array}{l}\text { UGT1A8 c.518GG genotype } \\
\text { was associated with higher } \\
\text { gastrointestinal symptom } \\
\text { rating scale (GSRS) score } \\
\text { up two weeks }\end{array}$ & [41] \\
\hline & & c.518G: $25 \%$ & $\begin{array}{l}\text { Prospective, multicenter } \\
\text { (Dominos study) }\end{array}$ & $\begin{array}{l}189 \text { adult patients } \\
\text { (France) }\end{array}$ & $\begin{array}{l}\text { EC-MPS } \\
\text { Cyclosporine } \\
\text { Corticosteroids } \\
\text { Six-month follow-up }\end{array}$ & - & $\begin{array}{l}\text { No association of } U G T 1 A 8 \\
\text { c. } 518 C>G \text { variant with } A R \\
\text { or adverse events }\end{array}$ & [29] \\
\hline & & c. $518 \mathrm{G}: 52 \%$ & Prospective & $\begin{array}{l}127 \text { adult patients } \\
\text { (China) }\end{array}$ & $\begin{array}{l}\text { MMF } \\
\text { Tacrolimus } \\
\text { Corticosteroids }\end{array}$ & $\begin{array}{l}\text { UGT1A8 c.518G allele } \\
\text { was associated with } \\
\text { lower MPAG AUC }\end{array}$ & - & [35] \\
\hline & & - & $\begin{array}{l}\text { Retrospective (Case- } \\
\text { Control), multicenter }\end{array}$ & $\begin{array}{l}284 \text { pediatric and young } \\
\text { adult patients (USA) }\end{array}$ & $\begin{array}{l}\text { MMF } \\
\text { Cyclosporine } \\
\text { Tacrolimus } \\
\text { Sirolimus } \\
\text { Corticosteroids } \\
\text { One-year follow-up }\end{array}$ & - & $\begin{array}{l}\text { No association of } \\
U G T 1 A 8^{*} 2 \text { and } U G T 1 A 8^{*} 3 \\
\text { variants with leukopenia }\end{array}$ & [27] \\
\hline & & c.518G: $49 \%$ & Retrospective & $\begin{array}{l}408 \text { adult patients } \\
\text { (China) }\end{array}$ & $\begin{array}{l}\text { MMF } \\
\text { Cyclosporine Tacrolimus } \\
\text { Corticosteroids } \\
\text { 40-day follow-up }\end{array}$ & $\begin{array}{l}\text { No association of } \\
\text { UGT1A8 c.518C }>\text { G } \\
\text { variant with MPA C/D } \\
\text { (days 3-8) }\end{array}$ & - & [38] \\
\hline
\end{tabular}




\begin{tabular}{|c|c|c|c|c|c|c|c|c|}
\hline Gene & Variant & Allele frequency & Study design & Population & $\begin{array}{c}\text { Immunosuppressive } \\
\text { regimen }\end{array}$ & Pharmacokinetics & Clinical outcomes & Ref. \\
\hline \multirow[t]{9}{*}{ UGTIA9 } & $\begin{array}{l}r s 17868320 \\
(c .-2152 C>T) \\
r s 6731242 \\
(-1887 T>G) \\
r s 13418420 \\
(-1818 T>C)\end{array}$ & $\begin{array}{l}\text { c.-2152T: } 7 \% \\
\text { c. }-275 \mathrm{~A}: 9 \% \\
\text { c.98C: } 2 \%\end{array}$ & Prospective & $\begin{array}{l}95 \text { adult patients } \\
\text { (Belgium) }\end{array}$ & $\begin{array}{l}\text { MMF } \\
\text { Tacrolimus } \\
\text { Corticosteroids } \\
\text { One-year follow-up }\end{array}$ & $\begin{array}{l}\text { Treatment with MMF } \\
2 \mathrm{~g}(n=32) \text { and } \\
\text { UGT1A9 c.-2152T and/ } \\
\text { or c.-275A alleles were } \\
\text { associated with MPA } \\
\text { pharmacokinetics, } \\
\text { including lower AUC } C_{0-} \\
12 \text { and 6-12, at } 7 \text { days post- } \\
\text { transplantation }\end{array}$ & $\begin{array}{l}\text { No association of UGT1A9 } \\
\text { c. }-2152 C>T, \text { c. }-275 T>A \text { or } \\
\text { c. } 98 T>C \text { variants with AR } \\
\text { or adverse events }\end{array}$ & [47] \\
\hline & $\begin{array}{l}r s 10176426 \\
(-665 C>T) \\
r s 2741045 \\
(c .-440 C>T) \\
r s 2741046 \\
(c .-331 T>C)\end{array}$ & $\begin{array}{l}\text { c.-2152T: } 1 \% \\
\text {-1887G: } 13 \% \\
-665 T: 4 \% \\
-440 /-331 \text { T/C: } 41 \% \\
\text { c.-275A: } 5 \% \\
\text { c.98C: } 0 \%\end{array}$ & Mixed & $\begin{array}{l}40 \text { adult patients } \\
\text { (Italy) }\end{array}$ & $\begin{array}{l}\text { MMF } \\
\text { Cyclosporine } \\
\text { Corticosteroid-free } \\
\text { At Month } 6\end{array}$ & $\begin{array}{l}\text { UGT1A9 - } 665 C T \\
\text { genotype was associated } \\
\text { with higher MPA C/D } \\
\text { and c.-440/c.-331TT/ } \\
\text { CC genotypes were } \\
\text { associated with higher } \\
\text { MPA exposure } \\
\left(A \cup C_{0-2,0-4 \text { and } 0-12} \text { ) }\right.\end{array}$ & $\begin{array}{l}\text { No association of } U G T 1 A 9 \\
\text { c. }-440 C>T \text { (or c. }-331 T>C \text { ) } \\
\text { variant with gastrointestinal } \\
\text { adverse events }\end{array}$ & [48] \\
\hline & $\begin{array}{l}\text { rs6714486 } \\
\text { (c.-275T>A) }\end{array}$ & I399C: 36\% & Prospective & 80 adult patients (Japan) & $\begin{array}{l}\text { MMF } \\
\text { Tacrolimus } \\
\text { Corticosteroids } \\
\text { At day } 28\end{array}$ & $\begin{array}{l}\text { No association of } \\
\text { UGT1A9 I399T }>C \\
\text { variant with MPA } \\
\text { pharmacokinetics }\end{array}$ & - & [33] \\
\hline & $\begin{array}{l}\text { rs3832043 } \\
\text { (-118delT) }\end{array}$ & - & Prospective & $\begin{array}{l}100 \text { adult patients } \\
\text { (Belgium) }\end{array}$ & $\begin{array}{l}\text { MMF } \\
\text { Tacrolimus }\end{array}$ & $\begin{array}{l}\text { UGT1A9 c. }-275 \mathrm{~T}>\mathrm{A} \text { and/ } \\
\text { or c. }-2152 \mathrm{C}>\mathrm{T} \text { variant }\end{array}$ & & [45] \\
\hline & $\begin{array}{l}\text { rs72551330 } \\
(\text { UGT1A9*3, c.98T>C) }\end{array}$ & & & & $\begin{array}{l}\text { Corticosteroids } \\
\text { Five-year follow-up }\end{array}$ & $\begin{array}{l}\text { was associated with low } \\
\text { MPA exposure }\left(\text { AUC }_{0-12}\right. \\
\left.<30 \mathrm{mg} / \mathrm{L} \text {. } \mathrm{h}^{-1}\right)\end{array}$ & & \\
\hline & $\begin{array}{l}\text { rs } 2741049 \\
(1399 \mathrm{~T}>\mathrm{C})\end{array}$ & & & & & & & \\
\hline & & $\begin{array}{l}\text { c.-2152T: } 9 \% \\
-331 \text { C: } 28 \% \\
\text { c.-275A: } 11 \% \\
\text { c. } 98 \text { C: } 0 \%\end{array}$ & $\begin{array}{l}\text { Retrospective (Case- } \\
\text { Control) }\end{array}$ & $\begin{array}{l}38 \text { pediatric patients } \\
\text { (USA) }\end{array}$ & $\begin{array}{l}\text { MMF } \\
\text { Cyclosporine } \\
\text { Tacrolimus } \\
\text { Corticosteroids }\end{array}$ & - & $\begin{array}{l}\text { UGT1A9 c.-331C allele } \\
\text { was associated with } \\
\text { adverse events (especially } \\
\text { leukopenia) }\end{array}$ & [43] \\
\hline & & $\begin{array}{l}\text { c. }-2152 T: 5 \% \\
\text { c. }-275 \mathrm{~A}: 6 \% \\
\text { c.98C: } 2 \% \\
\text { I399T: } 38 \%\end{array}$ & $\begin{array}{l}\text { Prospective, multicenter } \\
\text { (FDCC Study) }\end{array}$ & 338 adult patients & $\begin{array}{l}\text { MMF } \\
\text { Cyclosporine } \\
\text { Tacrolimus } \\
\text { Corticosteroids } \\
\text { One-year follow-up }\end{array}$ & $\begin{array}{l}\text { UGT1A9 C.-2152CT and } \\
\text { c.-275TA genotypes } \\
\text { were associated with } \\
\text { lower MPA AUC } C_{0-12} \text {, on } \\
\text { tacrolimus treatment, } \\
\text { especially at day } 3 \text {. } \\
\text { c.98TC genotype was } \\
\text { associated with higher } \\
\text { AUC }_{0-12}\end{array}$ & $\begin{array}{l}\text { UGT1A9 c.-2152CT and/ } \\
\text { or c.-275TA genotypes was } \\
\text { associated with increased } \\
\text { risk for AR, on tacrolimus } \\
\text { treatment }\end{array}$ & [39] \\
\hline & & - & $\begin{array}{l}\text { Mixed } \\
\text { (Case-control) }\end{array}$ & $\begin{array}{l}133 \text { adult patients }- \text { for } \\
\text { PK profile } n=30 \text { (Spain) }\end{array}$ & $\begin{array}{l}\text { MMF } \\
\text { EC-MPS } \\
\text { Tacrolimus }\end{array}$ & 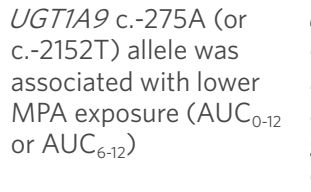 & $\begin{array}{l}\text { UGT1A9 c.-275A (or } \\
\text { c.-2152T) allele was } \\
\text { associated with more } \\
\text { admissions due to } \\
\text { gastrointestinal adverse } \\
\text { events }\end{array}$ & [46] \\
\hline
\end{tabular}




\begin{tabular}{|c|c|c|c|c|c|c|c|c|}
\hline Gene & Variant & Allele frequency & Study design & Population & $\begin{array}{c}\text { Immunosuppressive } \\
\text { regimen }\end{array}$ & Pharmacokinetics & Clinical outcomes & Ref. \\
\hline & & $\begin{array}{l}\text { c. }-2152 \mathrm{~T}: 6 \% \\
\text { c. }-440 \mathrm{~T}: 24 \% \\
\text { c. }-275 \mathrm{~A}: 6 \% \\
\text { c. } 98 \mathrm{C}: 3 \%\end{array}$ & $\begin{array}{l}\text { Prospective (included } \\
\text { multicenter Apomygre } \\
\text { study) }\end{array}$ & $\begin{array}{l}185 \text { patients } \\
\text { (France) }\end{array}$ & $\begin{array}{l}\text { MMF } \\
\text { Cyclosporine } \\
\text { Tacrolimus } \\
\text { Sirolimus } \\
\text { Corticosteroids } \\
\text { At a point between 3-6 } \\
\text { months }\end{array}$ & $\begin{array}{l}\text { No association of } \\
\text { UGT1A9 c.-2152C }>T \text {, } \\
\text { c. }-440 C>T \text {, c. }-275 T>A \\
\text { and c.98T>C variants } \\
\text { with MPA or MPAG } \\
\text { pharmacokinetics }\end{array}$ & - & [14] \\
\hline & & c. $-275 A: 6 \%$ & Retrospective & $\begin{array}{l}256 \text { adult patients } \\
\text { (France) }\end{array}$ & $\begin{array}{l}\text { MMF } \\
\text { Cyclosporine Tacrolimus } \\
\text { Sirolimus } \\
\text { Up to } 115 \text { month follow- } \\
\text { up }\end{array}$ & - & $\begin{array}{l}\text { No association of UGT1A9 } \\
\text { c.- } 275 T>A \text { variant with } \\
\text { gastrointestinal adverse } \\
\text { events }\end{array}$ & [34] \\
\hline & & $\begin{array}{l}\text { c. }-2152 \mathrm{~T}: 4 \% \\
\text { c. }-275 \mathrm{~A}: 6 \% \\
\text { c. } 98 \mathrm{C}: 2 \%\end{array}$ & Prospective, multicenter & $\begin{array}{l}89 \text { pediatric patients } \\
\text { (France) }\end{array}$ & $\begin{array}{l}\text { MMF } \\
\text { Cyclosporine } \\
\text { Tacrolimus } \\
\text { Corticosteroids Sixty-day } \\
\text { follow-up }\end{array}$ & $\begin{array}{l}\text { No association of } \\
\text { UGT1A9 c.-2152C }>\text { T, c.- } \\
275 T>A \text { and c. } 98 T>C \\
\text { variants with MPA } \\
\text { pharmacokinetics }\end{array}$ & - & [40] \\
\hline & & $\begin{array}{l}\text { c. }-275 \mathrm{~A}: 7 \% \\
\text { c. } 98 \mathrm{C}: 1 \%\end{array}$ & $\begin{array}{l}\text { Retrospective } \\
\text { (Transgene study) }\end{array}$ & $\begin{array}{l}218 \text { adult patients } \\
\text { (France) }\end{array}$ & $\begin{array}{l}\text { MMF } \\
\text { Cyclosporine } \\
\text { Tacrolimus } \\
\text { mTOR inhibitor } \\
\text { Corticosteroids } \\
\text { One-year follow-up }\end{array}$ & - & $\begin{array}{l}\text { No association of UGT1A9 } \\
\text { c. }-275 T>A \text { and c. } 98 T>C \\
\text { variants with AR or adverse } \\
\text { events }\end{array}$ & {$[13]$} \\
\hline & & $\begin{array}{l}\text { c. }-2152 \text { T: } 5 \% \\
\text { c. }-275 \mathrm{~A}: 4 \% \\
\text { c. } 98 \mathrm{C}: 3 \%\end{array}$ & $\begin{array}{l}\text { Retrospective (Case- } \\
\text { control) }\end{array}$ & $\begin{array}{l}103 \text { adult patients } \\
\text { (Poland) }\end{array}$ & $\begin{array}{l}\text { MMF } \\
\text { Calcineurin inhibitors } \\
\text { Corticosteroids } \\
\text { One-year follow-up }\end{array}$ & - & $\begin{array}{l}\text { UGT1A9 c.98TC genotype } \\
\text { was associated with } \\
\text { decreased graft function }\end{array}$ & [51] \\
\hline & & c.98C: $4 \%$ & Retrospective & $\begin{array}{l}140 \text { adult patients } \\
\text { (Poland) }\end{array}$ & $\begin{array}{l}\text { MMF } \\
\text { Cyclosporine } \\
\text { Corticosteroids }\end{array}$ & - & $\begin{array}{l}\text { UGT1A9 c.98TC genotype } \\
\text { was associated with lower } \\
\text { eGFR, but not with AR and } \\
\text { DGF }\end{array}$ & [52] \\
\hline & & $\begin{array}{l}\text { c.-2152T: } 3 \% \\
\text { c. }-275 \mathrm{~A}: 5 \%\end{array}$ & $\begin{array}{l}\text { Prospective, multicenter } \\
\text { (Dominos study) }\end{array}$ & $\begin{array}{l}189 \text { adult patients } \\
\text { (France) }\end{array}$ & $\begin{array}{l}\text { EC-MPS } \\
\text { Cyclosporine } \\
\text { Corticosteroids } \\
\text { Six-month follow-up }\end{array}$ & - & $\begin{array}{l}\text { No association of UGT1A9 } \\
\text { c. }-2152 C>T \text { and c. }-275 T>A \\
\text { variants with AR or adverse } \\
\text { events }\end{array}$ & [29] \\
\hline & & $\begin{array}{l}\text {-1818T: } 13 \% \\
\text { C.-440T: } 2 \% \\
\text { C.-331C: } 2 \% \\
\text {-118delT: } 58 \% \\
\text { I399C: } 31 \%\end{array}$ & Prospective & $\begin{array}{l}127 \text { adult patients } \\
\text { (China) }\end{array}$ & $\begin{array}{l}\text { MMF } \\
\text { Tacrolimus } \\
\text { Corticosteroids }\end{array}$ & $\begin{array}{l}\text { UGT1A9 c.-440CT } \\
\text { (or c.-331TC) and } \\
\text {-1818CC genotypes were } \\
\text { associated with higher } \\
\text { and lower MPAG } \\
\text { AUC }_{0-12} \text {, respectively }\end{array}$ & - & [35] \\
\hline & & - & $\begin{array}{l}\text { Retrospective (Case- } \\
\text { control) multicenter }\end{array}$ & $\begin{array}{l}284 \text { pediatric and young } \\
\text { adult patients (USA) }\end{array}$ & $\begin{array}{l}\text { MMF } \\
\text { Cyclosporine } \\
\text { Tacrolimus } \\
\text { Sirolimus } \\
\text { Corticosteroids } \\
\text { One-year follow-up }\end{array}$ & - & $\begin{array}{l}\text { No association of UGT1A9 } \\
\text { c. }-2152 \mathrm{C}>\mathrm{T},-665 \mathrm{C}>\mathrm{T}, \mathrm{c} .- \\
440 \mathrm{C}>\mathrm{T} \text { and } \mathrm{c} .-275 \mathrm{~T}>\mathrm{A} \\
\text { variants with leukopenia }\end{array}$ & [27] \\
\hline
\end{tabular}




\begin{tabular}{|c|c|c|c|c|c|c|c|c|}
\hline Gene & Variant & Allele frequency & Study design & Population & $\begin{array}{c}\text { Immunosuppressive } \\
\text { regimen }\end{array}$ & Pharmacokinetics & Clinical outcomes & Ref. \\
\hline & & - & Retrospective & $\begin{array}{l}118 \text { adult patients } \\
\text { (China) }\end{array}$ & $\begin{array}{l}\text { MMF } \\
\text { Cyclosporine } \\
\text { Tacrolimus } \\
\text { Corticosteroids }\end{array}$ & $\begin{array}{l}\text { No association of } \\
\text { UGT1A9 -118delT } \\
\text { variant with MPA } \\
\text { pharmacokinetics }\end{array}$ & -- & [50] \\
\hline & & - & $\begin{array}{l}\text { Retrospective (Case- } \\
\text { control) }\end{array}$ & $\begin{array}{l}145 \text { adult patients } \\
\text { (Brazil) }\end{array}$ & $\begin{array}{l}\text { MMF } \\
\text { Cyclosporine Tacrolimus } \\
\text { Sirolimus } \\
\text { Corticosteroids }\end{array}$ & - & $\begin{array}{l}\text { No association of UGT1A9 } \\
\text { c. }-275 T>A \text { variant with AR }\end{array}$ & {$[22]$} \\
\hline & & $\begin{array}{l}\text { c. }-275 \text { A: } 14 \% \\
\text { c. } 98 \text { C: } 0 \%\end{array}$ & Prospective & $\begin{array}{l}21 \text { adult patients } \\
\text { (Canada) }\end{array}$ & $\begin{array}{l}\text { MMF } \\
\text { Tacrolimus } \\
\text { Corticosteroid-free } \\
\text { One-year follow-up }\end{array}$ & $\begin{array}{l}\text { No association of } \\
\text { UGT1A9 c.- } 275 \mathrm{~T}>\mathrm{A} \text { and } \\
\text { c. } 98 \mathrm{~T}>\mathrm{C} \text { variants with } \\
\text { MPA exposure (AUC) }\end{array}$ & $\begin{array}{l}\text { No association of UGTIA9 } \\
\text { c. }-275 T>A \text { and c. } 98 \mathrm{~T}>\mathrm{C} \\
\text { variants with neutrophil } \\
\text { counts }\end{array}$ & [49] \\
\hline & & $\begin{array}{l}\text { c. }-440 \mathrm{~T}: 3 \% \\
\text { c. } 331 \text { C: } 3 \% \\
\text { c. } 1399 \text { C: } 47 \%\end{array}$ & Retrospective & $\begin{array}{l}408 \text { adult patients } \\
\text { (China) }\end{array}$ & $\begin{array}{l}\text { MMF } \\
\text { Cyclosporine } \\
\text { Tacrolimus } \\
\text { Corticosteroids } \\
\text { 40-day follow-up }\end{array}$ & $\begin{array}{l}\text { No association of } \\
\text { UGT1A9 c.- } 440 C>T \text {, c.- } \\
331 T>C \text { and I399 T }>C \\
\text { variants with MPA C/D } \\
\text { (days 3-8) }\end{array}$ & - & [38] \\
\hline & rs6744284 & rs6744284T: $32 \%$ & Prospective & $\begin{array}{l}109 \text { adult patients } \\
\text { (USA) }\end{array}$ & $\begin{array}{l}\text { MMF } \\
\text { EC-MPS } \\
\text { Cyclosporine } \\
\text { Tacrolimus } \\
\text { Corticosteroids } \\
\text { Six-month follow-up }\end{array}$ & - & $\begin{array}{l}\text { UGT1A9 rs } 6744284 C C \\
\text { genotype was associated } \\
\text { with higher gastrointestinal } \\
\text { symptom rating scale } \\
\text { (GSRS) score for } \\
\text { constipation at week } 1\end{array}$ & {$[41]$} \\
\hline \multirow[t]{5}{*}{ UGT2B7 } & $\begin{array}{l}\text { rs } 7438135 \\
\text { (c. }-840 \mathrm{G}>\mathrm{A}, \\
\text { c. }-842 \mathrm{G}>\mathrm{A} \text { or c.- } \\
900 \mathrm{G}>\mathrm{A}) \\
\text { rs } 7662029 \\
(\mathrm{c} .-327 \mathrm{G}>\mathrm{A})\end{array}$ & $\begin{array}{l}\text { C. }-842 \mathrm{G}: 55 \% \\
802 \mathrm{~T}: 55 \%\end{array}$ & Prospective, multicenter & $\begin{array}{l}92 \text { adult patients } \\
\text { (France) }\end{array}$ & $\begin{array}{l}\text { MMF } \\
\text { Cyclosporine } \\
\text { Tacrolimus } \\
\text { Sirolimus } \\
\text { Corticosteroids Three- } \\
\text { month follow-up }\end{array}$ & $\begin{array}{l}\text { UGT2B7 c.-842AA } \\
\text { ( } 802 C C \text { ) genotype was } \\
\text { associated with higher } \\
\text { AcMPAG AUC } \text { C.-9 } \\
\text { at Month } 1 \text { and } 3 \text {, on } \\
\text { sirolimus treatment ( } n= \\
\text { 40) }\end{array}$ & - & {$[53]$} \\
\hline & $\begin{array}{l}\text { rs73823859 } \\
(-79 G>A) \\
\text { rs12233719 }\end{array}$ & $802 \mathrm{~T}: 41 \%$ & Mixed & $\begin{array}{l}40 \text { adult patients } \\
\text { (Italy) }\end{array}$ & $\begin{array}{l}\text { MMF } \\
\text { Cyclosporine } \\
\text { Corticosteroid-free } \\
\text { At Month } 6\end{array}$ & $\begin{array}{l}\text { UGT2B7 802TT } \\
\text { genotype individuals } \\
\text { showed higher MPA peak } \\
\text { concentration than those } \\
\text { with } 802 C \text { genotype }\end{array}$ & - & [48] \\
\hline & $\begin{array}{l}(c .2 T T G>1) \\
\text { rs7439366 } \\
\left(802 C>T, U G T 2 B 7^{\star} 2\right)\end{array}$ & UGT2B7*2(T): $33 \%$ & Prospective & 72 adult patients (Japan) & $\begin{array}{l}\text { MMF } \\
\text { Tacrolimus } \\
\text { Corticosteroids } \\
\text { At day } 28\end{array}$ & $\begin{array}{l}\text { No association of } \\
\text { UGT2B } 7^{\star} 2 \text { variant with } \\
\text { daytime or nighttime } \\
\text { MPA pharmacokinetics }\end{array}$ & - & [36] \\
\hline & $\begin{array}{l}\text { rs62298861 } \\
(\text { IVS1+985A>G) }\end{array}$ & c. $-840 \mathrm{G}: 50 \%$ & $\begin{array}{l}\text { Prospective } \\
\text { (FDCC } \\
\text { Study) }\end{array}$ & 332 adult patients & $\begin{array}{l}\text { MMF } \\
\text { Cyclosporine } \\
\text { Tacrolimus } \\
\text { Corticosteroid } \\
\text { One-year follow-up }\end{array}$ & $\begin{array}{l}\text { No association of } \\
\text { UGT2B7 c.-840G>A } \\
\text { variant with exposure } \\
\text { to AcMPAG (level and } \\
\text { AUCs) }\end{array}$ & $\begin{array}{l}\text { No association of } \\
\text { UGT2B7c.-840G }>\text { A variant } \\
\text { with diarrhea or leukopenia }\end{array}$ & [54] \\
\hline & & c. $-900 \mathrm{G}: 45 \%$ & $\begin{array}{l}\text { Retrospective (Case- } \\
\text { control) }\end{array}$ & $\begin{array}{l}38 \text { pediatric patients } \\
\text { (USA) }\end{array}$ & $\begin{array}{l}\text { MMF } \\
\text { Cyclosporine } \\
\text { Tacrolimus } \\
\text { Corticosteroids }\end{array}$ & - & $\begin{array}{l}\text { No association of UGT2BT } \\
\text { c. }-900 A>G \text { variant with } \\
\text { adverse events (diarrhea } \\
\text { and leukopenia) }\end{array}$ & [43] \\
\hline
\end{tabular}




\begin{tabular}{|c|c|c|c|c|c|c|c|c|}
\hline Gene & Variant & Allele frequency & Study design & Population & $\begin{array}{c}\text { Immunosuppressive } \\
\text { regimen }\end{array}$ & Pharmacokinetics & Clinical outcomes & Ref. \\
\hline & & $\begin{array}{l}\text { c.- }-842 A: 50 \% \\
-79 A: 2 \% \\
\text { 802C: } 50 \%\end{array}$ & $\begin{array}{l}\text { Prospective, multicenter } \\
\text { (FDCC } \\
\text { Study) }\end{array}$ & 338 adult patients & $\begin{array}{l}\text { MMF } \\
\text { Cyclosporine } \\
\text { Tacrolimus } \\
\text { Corticosteroids } \\
\text { One-year follow-up }\end{array}$ & $\begin{array}{l}\text { No association of } \\
U G T 2 B 7 \text { C.-842G }>A \text {, } \\
-79 G>A \text { and } 802 C>T \\
\text { variants with MPA } \\
\text { exposure }\left(A \cup C_{0-12}\right)\end{array}$ & $\begin{array}{l}\text { No association of } U G T 2 B 7 \\
\text { C.- }-842 G>A,-79 G>A \text { and } \\
802 C>T \text { variants with } A R\end{array}$ & [39] \\
\hline & & c. $-840 \mathrm{~A}: 48 \%$ & Retrospective & $\begin{array}{l}256 \text { adult patients } \\
\text { (France) }\end{array}$ & $\begin{array}{l}\text { MMF } \\
\text { Cyclosporine } \\
\text { Tacrolimus } \\
\text { Sirolimus } \\
\text { Up to 115-month follow- } \\
\text { up }\end{array}$ & - & $\begin{array}{l}\text { No association of } U G T 2 B 7 \\
\text { c.- } 840 G>A \text { variant with } \\
\text { gastrointestinal adverse } \\
\text { events }\end{array}$ & [34] \\
\hline & & $802 \mathrm{~T}: 48 \%$ & Prospective, multicenter & $\begin{array}{l}89 \text { pediatric patients } \\
\text { (France) }\end{array}$ & $\begin{array}{l}\text { MMF } \\
\text { Cyclosporine } \\
\text { Tacrolimus } \\
\text { Corticosteroids Sixty-day } \\
\text { follow-up }\end{array}$ & $\begin{array}{l}\text { UGT2B7 802T allele was } \\
\text { associated with higher } \\
\text { MPA apparent oral } \\
\text { clearance }\end{array}$ & - & [40] \\
\hline & & $802 \mathrm{~T}: 46 \%$ & $\begin{array}{l}\text { Retrospective } \\
\text { (Transgene study) }\end{array}$ & $\begin{array}{l}218 \text { adult patients } \\
\text { (France) }\end{array}$ & $\begin{array}{l}\text { MMF } \\
\text { Cyclosporine } \\
\text { Tacrolimus } \\
\text { mTOR inhibitor } \\
\text { Corticosteroids } \\
\text { One-year follow-up }\end{array}$ & - & $\begin{array}{l}\text { No association of } U G T 2 B 7 \\
802 C>T \text { variant with AR or } \\
\text { adverse events }\end{array}$ & [13] \\
\hline & & c.-840G: 47\% & $\begin{array}{l}\text { Prospective, multicenter } \\
\text { (Dominos study) }\end{array}$ & $\begin{array}{l}189 \text { adult patients } \\
\text { (France) }\end{array}$ & $\begin{array}{l}\text { EC-MPS } \\
\text { Cyclosporine } \\
\text { Corticosteroids } \\
\text { Six-month follow-up }\end{array}$ & - & $\begin{array}{l}\text { UGT2B7 c.-840G allele } \\
\text { was associated with } \\
\text { increased risk of anemia. } \\
\text { No association with AR, } \\
\text { diarrhea, or leukopenia }\end{array}$ & [29] \\
\hline & & $\begin{array}{l}\text { c. }-900 \text { G: } 29 \% \\
\text { IVS1+985G: } 5 \% \\
\text { c. } 211 T: 14 \%\end{array}$ & Prospective & $\begin{array}{l}127 \text { adult patients } \\
\text { (China) }\end{array}$ & $\begin{array}{l}\text { MMF } \\
\text { Tacrolimus } \\
\text { Corticosteroids }\end{array}$ & $\begin{array}{l}\text { UGT2B7IVS1+985AG } \\
\text { genotype was associated } \\
\text { with higher MPA AUC }\end{array}$ & - & [35] \\
\hline & & - & $\begin{array}{l}\text { Retrospective (Case- } \\
\text { control) multicenter }\end{array}$ & $\begin{array}{l}284 \text { pediatric and young } \\
\text { adult patients (USA) }\end{array}$ & $\begin{array}{l}\text { MMF } \\
\text { Cyclosporine } \\
\text { Tacrolimus } \\
\text { Sirolimus } \\
\text { Corticosteroids } \\
\text { One-year follow-up }\end{array}$ & - & $\begin{array}{l}\text { In patients treated non- } \\
\text { depleting antibodies, } \\
\text { UGT2B7 c.- } 900 \mathrm{G} \text { allele was } \\
\text { associated with increased } \\
\text { risk of } \\
\text { leukopenia }\end{array}$ & [27] \\
\hline & & c.211T: 34\% & Retrospective & $\begin{array}{l}118 \text { adult patients } \\
\text { (China) }\end{array}$ & $\begin{array}{l}\text { MMF } \\
\text { Cyclosporine } \\
\text { Tacrolimus } \\
\text { Corticosteroids }\end{array}$ & $\begin{array}{l}\text { UGT2B } 7 \text { c. } 211 G>T \\
\text { variant was associated } \\
\text { with MPA initial volume } \\
\text { of distribution and } \\
\text { c. } 211 G T \text { genotype was } \\
\text { associated with higher } \\
\text { MPA exposure }\end{array}$ & - & [50] \\
\hline & & c. $-842 \mathrm{G}: 37 \%$ & $\begin{array}{l}\text { Retrospective (Case- } \\
\text { control) }\end{array}$ & $\begin{array}{l}145 \text { adult patients } \\
\text { (Brazil) }\end{array}$ & $\begin{array}{l}\text { MMF } \\
\text { Cyclosporine } \\
\text { Tacrolimus } \\
\text { Sirolimus } \\
\text { Corticosteroids }\end{array}$ & - & $\begin{array}{l}\text { UGT2B } 7 \text { C. }-842 \mathrm{G} \text { allele was } \\
\text { associated with prevention } \\
\text { of AR }\end{array}$ & [22] \\
\hline
\end{tabular}




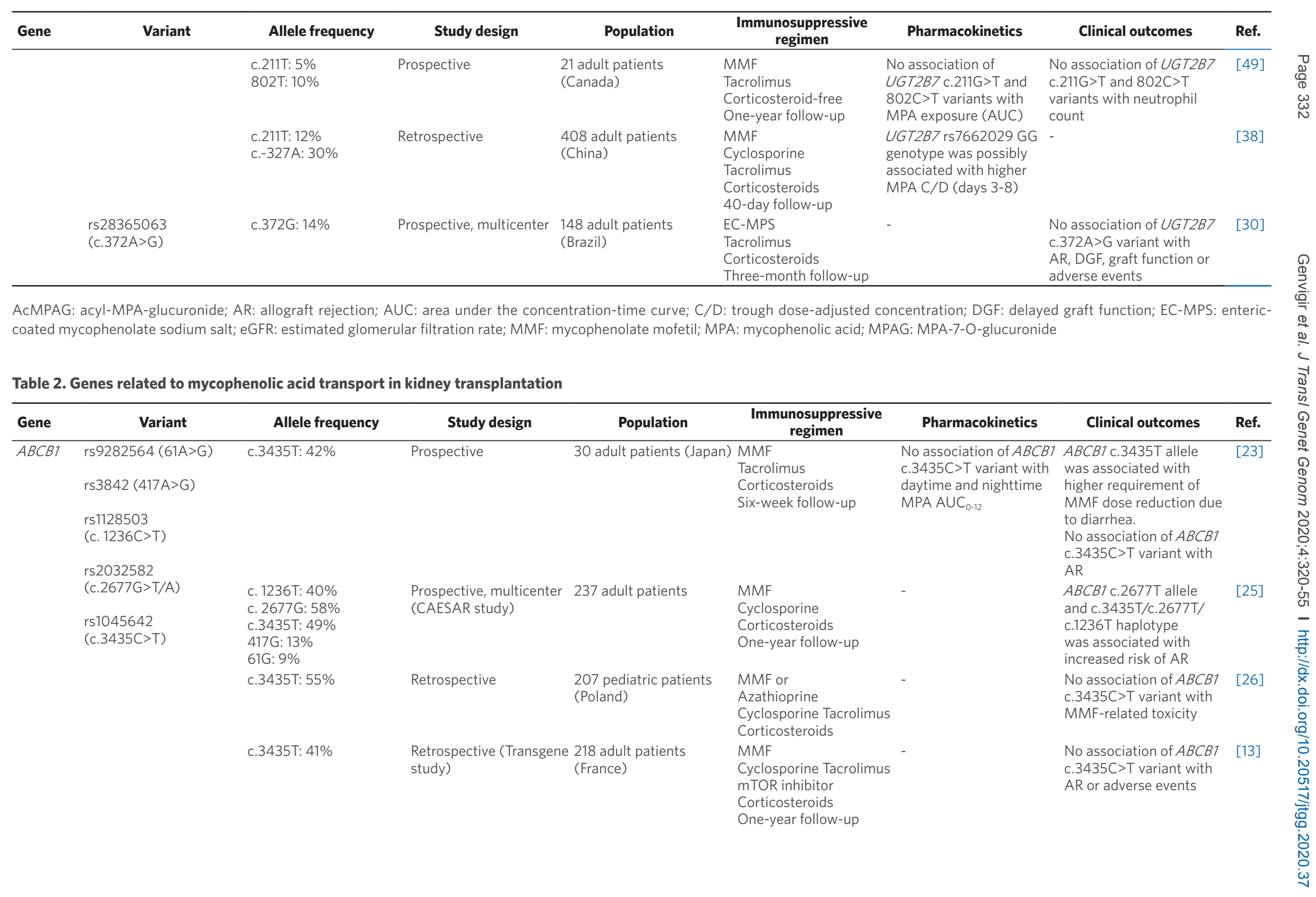




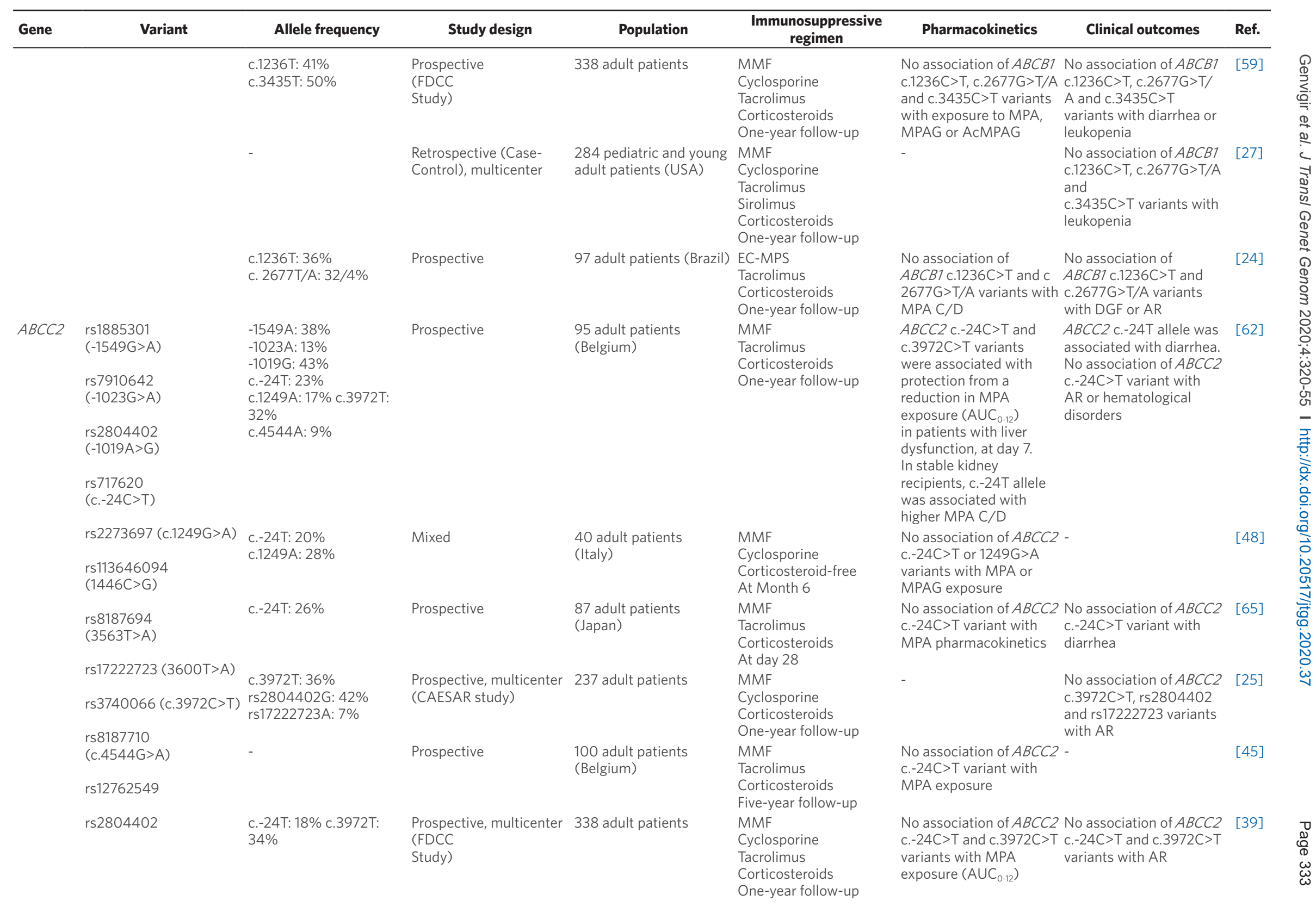




\begin{tabular}{|c|c|c|c|c|c|c|c|c|}
\hline Gene & Variant & Allele frequency & Study design & Population & $\begin{array}{c}\text { Immunosuppressive } \\
\text { regimen }\end{array}$ & Pharmacokinetics & Clinical outcomes & Ref. \\
\hline & & c.-24T: $23 \%$ & $\begin{array}{l}\text { Prospective (included } \\
\text { multicenter Apomygre } \\
\text { study) }\end{array}$ & $\begin{array}{l}185 \text { patients } \\
\text { (France) }\end{array}$ & $\begin{array}{l}\text { MMF } \\
\text { Cyclosporine } \\
\text { Tacrolimus } \\
\text { Sirolimus } \\
\text { Corticosteroids } \\
\text { At a point between 3-6 } \\
\text { months }\end{array}$ & $\begin{array}{l}\text { No association of } \\
A B C C 2 \text { c.- } 24 C>T \text { variant } \\
\text { with MPA or MPAG } \\
\text { pharmacokinetics }\end{array}$ & - & [14] \\
\hline & & $\begin{array}{l}\text { c. }-24 T: 24 \% \\
\text { c. } 3972 T: 39 \%\end{array}$ & Retrospective & $\begin{array}{l}256 \text { adult patients } \\
\text { (France) }\end{array}$ & $\begin{array}{l}\text { MMF } \\
\text { Cyclosporine Tacrolimus } \\
\text { Sirolimus } \\
\text { Up to } 115 \text {-month follow- } \\
\text { up }\end{array}$ & - & $\begin{array}{l}\text { No association of } \\
A B C C 2 \text { c. }-24 C>\text { T and } \\
\text { c. } 3972 C>\text { T variants with } \\
\text { gastrointestinal adverse } \\
\text { events }\end{array}$ & [34] \\
\hline & & $\begin{array}{l}\text { c.-24T:19\% c.1249A: 19\% } \\
\text { 3563A: } 10 \% \\
\text { c.3972T:35\% }\end{array}$ & Prospective, multicenter & $\begin{array}{l}89 \text { pediatric patients } \\
\text { (France) }\end{array}$ & $\begin{array}{l}\text { MMF } \\
\text { Cyclosporine } \\
\text { Tacrolimus } \\
\text { Corticosteroids } \\
\text { Sixty-day follow-up }\end{array}$ & $\begin{array}{l}\text { No association of } A B C C 2 \\
\text { c. }-24 C>\mathrm{T} \text {, c.1249G }>\mathrm{A} \text {, } \\
3563 \mathrm{~T}>\mathrm{A} \text { and } \\
\text { c.3972C }>\mathrm{T} \text { variants with } \\
\text { MPA pharmacokinetics }\end{array}$ & & [40] \\
\hline & & $\begin{array}{l}\text { c. }-24 \mathrm{~T}: 19 \% \\
\text { c.1249A: } 19 \% \\
\text { 1446G: } 0.7 \% \\
\text { c. } 3972 \mathrm{~T}: 35 \%\end{array}$ & $\begin{array}{l}\text { Retrospective (Transgene } \\
\text { study) }\end{array}$ & $\begin{array}{l}218 \text { adult patients } \\
\text { (France) }\end{array}$ & $\begin{array}{l}\text { MMF } \\
\text { Cyclosporine } \\
\text { Tacrolimus } \\
\text { mTOR inhibitor } \\
\text { Corticosteroids } \\
\text { One-year follow-up }\end{array}$ & - & $\begin{array}{l}\text { No association of } A B C C 2 \\
\text { c. }-24 C>T, c .1249 \mathrm{G}>\mathrm{A} \text {, } \\
1446 C>\mathrm{G} \text { and } \\
\text { c. } 3972 \mathrm{C}>\mathrm{T} \text { variants with } \\
\text { AR or adverse events }\end{array}$ & [13] \\
\hline & & $\begin{array}{l}\text { c.-24T: } 21 \% \\
\text { c. } 3972 \text { T: } 23 \%\end{array}$ & $\begin{array}{l}\text { Prospective, multicenter } \\
\text { (Symphony study) }\end{array}$ & $\begin{array}{l}55 \text { adult patients } \\
\text { (Spain) }\end{array}$ & $\begin{array}{l}\text { MMF } \\
\text { Cyclosporine } \\
\text { Tacrolimus } \\
\text { Sirolimus } \\
\text { Corticosteroids } \\
\text { Three-month follow-up }\end{array}$ & $\begin{array}{l}\text { ABCC2 } \mathrm{c} \text { - }-24 T \text { allele } \\
\text { was associated with } \\
\text { lower MPA AUC, on } \\
\text { cyclosporine-free } \\
\text { treatment }\end{array}$ & - & [63] \\
\hline & & c. $-24 \mathrm{~T}: 28 \%$ & $\begin{array}{l}\text { Prospective, } \\
\text { Multicenter } \\
\text { (Case-control) }\end{array}$ & $\begin{array}{l}37 \text { adult patients } \\
\text { (China) }\end{array}$ & $\begin{array}{l}\text { MMF } \\
\text { Cyclosporine Prednisone }\end{array}$ & \multicolumn{2}{|l|}{$\begin{array}{l}\text { No association of } A B C C 2 \text { - } \\
\text { c.- } 24 T \text { variant with MPA } \\
\text { or MPAG exposure } \\
\left(A \cup C_{4-12} \text { or } A \cup C_{0-12}\right)\end{array}$} & [37] \\
\hline & & $\begin{array}{l}\text { c. }-24 \mathrm{~T}: 19 \% \\
\text { c. } 3972 \mathrm{~T}: 36 \%\end{array}$ & $\begin{array}{l}\text { Prospective, multicenter } \\
\text { (Symphony study) }\end{array}$ & 56 adult patients & $\begin{array}{l}\text { MMF } \\
\text { Cyclosporine } \\
\text { Tacrolimus } \\
\text { Sirolimus } \\
\text { Corticosteroids } \\
\text { One-year follow-up }\end{array}$ & \multicolumn{2}{|l|}{$\begin{array}{l}\text { No association of } A B C C 2- \\
\text { c.-24C }>\mathrm{T} \text { and c.3972C }>\mathrm{T} \\
\text { variants with MPA (or its } \\
\text { metabolites) exposure }\end{array}$} & [66] \\
\hline & & $\begin{array}{l}\text { c. }-24 T: 17 \% \\
\text { c. } 1249 \text { A: } 21 \% \\
\text { c. } 3972 \text { T: } 39 \% \\
\text { c. } 4544 \text { A: } 8 \%\end{array}$ & $\begin{array}{l}\text { Prospective, multicenter } \\
\text { (Dominos study) }\end{array}$ & $\begin{array}{l}189 \text { adult patients } \\
\text { (France) }\end{array}$ & $\begin{array}{l}\text { EC-MPS } \\
\text { Cyclosporine } \\
\text { Corticosteroids } \\
\text { Six-month follow-up }\end{array}$ & 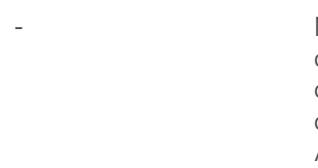 & $\begin{array}{l}\text { No association of } A B C C 2 \\
\text { c. }-24 C>T \text {, c. } 1249 \mathrm{G}>\mathrm{A} \text {, } \\
\text { c. } 3972 \mathrm{C}>\mathrm{T} \text { and } \\
\text { c. } 4544 \mathrm{G}>\mathrm{A} \text { variants with } \\
\text { AR or adverse events }\end{array}$ & [29] \\
\hline
\end{tabular}




\begin{tabular}{|c|c|c|c|c|c|c|c|c|}
\hline Gene & Variant & Allele frequency & Study design & Population & $\begin{array}{c}\text { Immunosuppressive } \\
\text { regimen }\end{array}$ & Pharmacokinetics & Clinical outcomes & Ref. \\
\hline & & $\begin{array}{l}\text { c. }-24 \mathrm{~T}: 14 \% \\
\text { c. } 3972 \mathrm{~T}: 32 \%\end{array}$ & Prospective, multicenter & $\begin{array}{l}148 \text { adult patients } \\
\text { (Brazil) }\end{array}$ & $\begin{array}{l}\text { EC-MPS } \\
\text { Tacrolimus } \\
\text { Corticosteroids } \\
\text { Three-month follow-up }\end{array}$ & 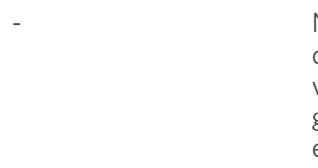 & $\begin{array}{l}\text { No association of } A B C C 2 \\
\text { c.- } 24 C>T \text { and } c .3972 C>T \\
\text { variants with } A R, D G F, \\
\text { graft function or adverse } \\
\text { events }\end{array}$ & [30] \\
\hline & & $\begin{array}{l}\text { c. }-24 \mathrm{~T}: 17 \% \\
\text { c.1249A: } 24 \%\end{array}$ & Prospective & $\begin{array}{l}68 \text { adult patients } \\
\text { (Croatia) }\end{array}$ & $\begin{array}{l}\text { MMF } \\
\text { EC-MPS } \\
\text { Cyclosporine } \\
\text { Tacrolimus } \\
\text { Corticosteroids }\end{array}$ & $\begin{array}{l}\text { Donors' } A B C C 2 \text { c.1249A } \\
\text { allele was associated } \\
\text { with a reduced peak and } \\
\text { early }\left(A \cup C_{0-2}\right) \text { exposure } \\
\text { to MPA }\end{array}$ & - & [64] \\
\hline & & - & $\begin{array}{l}\text { Retrospective (Case- } \\
\text { Control), multicenter }\end{array}$ & $\begin{array}{l}284 \text { pediatric and young } \\
\text { adult patients (USA) }\end{array}$ & $\begin{array}{l}\text { MMF } \\
\text { Cyclosporine } \\
\text { Tacrolimus } \\
\text { Sirolimus } \\
\text { Corticosteroids } \\
\text { One-year follow-up }\end{array}$ & - & $\begin{array}{l}\text { No association of } \\
A B C C 2 \text { c. }-24 C>T \text { and } \\
\text { rs } 12762549 \text { variants with } \\
\text { leukopenia }\end{array}$ & [27] \\
\hline & & $\begin{array}{l}\text { c.-24T: } 20 \% \\
\text { c.1249A: } 22 \%\end{array}$ & $\begin{array}{l}\text { Retrospective (Case- } \\
\text { control) }\end{array}$ & $\begin{array}{l}145 \text { adult patients } \\
\text { (Brazil) }\end{array}$ & $\begin{array}{l}\text { MMF } \\
\text { Cyclosporine } \\
\text { Tacrolimus } \\
\text { Sirolimus } \\
\text { Corticosteroids }\end{array}$ & i & $\begin{array}{l}\text { No association of } A B C C 2 \\
\text { c.-24C>T and c. } 1249 \mathrm{G}>\mathrm{A} \\
\text { variants with } A R\end{array}$ & [22] \\
\hline & & $\begin{array}{l}\text { c.1249A: } 19 \% \\
\text { c.3972T: } 30 \%\end{array}$ & Prospective & 97 adult patients (Brazil) & $\begin{array}{l}\text { EC-MPS } \\
\text { Tacrolimus } \\
\text { Corticosteroids } \\
\text { One-year follow-up }\end{array}$ & $\begin{array}{l}\text { No association of } \\
\text { ABCC } 2 \text { c. } 1249 \mathrm{G}>\mathrm{A} \text { and } \\
\text { c. } 3972 \mathrm{C}>\mathrm{T} \text { variants with } \\
\text { MPA C/D }\end{array}$ & $\begin{array}{l}\text { No association of } \\
A B C C 2 \text { c. } 1249 \mathrm{G}>\mathrm{A} \text { and } \\
\text { c. } 3972 \mathrm{C}>\mathrm{T} \text { variants with } \\
\text { DGF or } \mathrm{AR}\end{array}$ & [24] \\
\hline & & $\begin{array}{l}\text { c.-24T: } 19 \% \\
\text { c.1249A: } 0 \%\end{array}$ & Prospective & $\begin{array}{l}21 \text { adult patients } \\
\text { (Canada) }\end{array}$ & $\begin{array}{l}\text { MMF } \\
\text { Tacrolimus } \\
\text { Corticosteroid-free } \\
\text { One-year follow-up }\end{array}$ & $\begin{array}{l}\text { No association of } A B C C 2 \\
\text { c. }-24 C>T \text { and c. } 1249 \mathrm{G}>\mathrm{A} \\
\text { variants with MPA } \\
\text { exposure (AUC) }\end{array}$ & $\begin{array}{l}\text { No association of } A B C C 2 \\
\text { c. }-24 C>T \text { and c. } 1249 \mathrm{G}>\mathrm{A} \\
\text { variants with neutrophil } \\
\text { counts }\end{array}$ & [49] \\
\hline & & c.-24T: $21 \%$ c.1249A: $8 \%$ & Retrospective & $\begin{array}{l}408 \text { adult patients } \\
\text { (China) }\end{array}$ & $\begin{array}{l}\text { MMF } \\
\text { Cyclosporine } \\
\text { Tacrolimus } \\
\text { Corticosteroids } \\
\text { 40-day follow-up }\end{array}$ & $\begin{array}{l}\text { ABCC2 c.-24TT } \\
\text { genotype was possibly } \\
\text { associated with higher } \\
\text { MPA C/D (days 3-8) }\end{array}$ & - & [38] \\
\hline \multirow[t]{3}{*}{$A B C G 2$} & $\begin{array}{l}r s 2231142 \\
(c .421 C>A) \\
r s 4491984 G>A\end{array}$ & c. $421 \mathrm{~A}: 26 \%$ & $\begin{array}{l}\text { Prospective, } \\
\text { Multicenter } \\
\text { (Case-control) }\end{array}$ & $\begin{array}{l}37 \text { adult patients } \\
\text { (China) }\end{array}$ & $\begin{array}{l}\text { MMF } \\
\text { Cyclosporine } \\
\text { Corticosteroids }\end{array}$ & $\begin{array}{l}\text { No association of } A B C G 2 \\
\text { c. } 421 C>A \text { variant with } \\
\text { MPA or MPAG exposure } \\
\left(A \cup C_{4-12} \text { or } A \cup C_{0-12}\right)\end{array}$ & & [37] \\
\hline & & c. $421 \mathrm{~A}: 8 \%$ & Prospective, multicenter & $\begin{array}{l}148 \text { adult patients } \\
\text { (Brazil) }\end{array}$ & $\begin{array}{l}\text { EC-MPS } \\
\text { Tacrolimus } \\
\text { Corticosteroids } \\
\text { Three-month follow-up }\end{array}$ & - & $\begin{array}{l}\text { No association of } A B C G 2 \\
\text { c. } 421 C>A \text { variant with } \\
\text { AR, DGF, graft function } \\
\text { or adverse events }\end{array}$ & [30] \\
\hline & & - & $\begin{array}{l}\text { Retrospective (Case- } \\
\text { Control), multicenter }\end{array}$ & $\begin{array}{l}284 \text { pediatric and young } \\
\text { adult patients (USA) }\end{array}$ & $\begin{array}{l}\text { MMF } \\
\text { Cyclosporine } \\
\text { Tacrolimus } \\
\text { Sirolimus } \\
\text { Corticosteroids } \\
\text { One-year follow-up }\end{array}$ & i & $\begin{array}{l}\text { No association of } A B C G 2 \\
\text { c. } 421 C>A \\
\text { variant with leukopenia }\end{array}$ & [27] \\
\hline
\end{tabular}




\begin{tabular}{|c|c|c|c|c|c|c|c|c|}
\hline Gene & Variant & Allele frequency & Study design & Population & $\begin{array}{c}\text { Immunosuppressive } \\
\text { regimen }\end{array}$ & Pharmacokinetics & Clinical outcomes & Ref. \\
\hline & & c. $421 \mathrm{~A}: 10 \%$ & Prospective & $\begin{array}{l}97 \text { adult patients } \\
\text { (Brazil) }\end{array}$ & $\begin{array}{l}\text { EC-MPS } \\
\text { Tacrolimus } \\
\text { Corticosteroids One-year } \\
\text { follow-up }\end{array}$ & $\begin{array}{l}\text { No association of } A B C G 2 \\
\text { c. } 421 C>A \text { variant with } \\
\text { MPA C/D }\end{array}$ & $\begin{array}{l}2 \text { No association of } A B C G 2 \\
\text { c. } 421 C>A \text { variant with } \\
\text { DGF or AR }\end{array}$ & [24] \\
\hline & & $\begin{array}{l}\text { c.421A: 31\% } \\
\text { rs4491984A: 50\% }\end{array}$ & Retrospective & $\begin{array}{l}408 \text { adult patients } \\
\text { (China) }\end{array}$ & $\begin{array}{l}\text { MMF } \\
\text { Cyclosporine } \\
\text { Tacrolimus } \\
\text { Corticosteroids } \\
\text { 40-day follow-up }\end{array}$ & $\begin{array}{l}\text { No association of } \\
\text { ABCG2 c. } 421 C>A \text { and } \\
\text { rs } 4491984 \text { variants with } \\
\text { MPA C/D (days 3-8) }\end{array}$ & - & [38] \\
\hline \multirow[t]{8}{*}{ SLCO1B1 } & $\begin{array}{l}\text { rs4149015 } \\
(-11187 G>A) \\
\text { rs2306283 }(c .388 A>G)\end{array}$ & 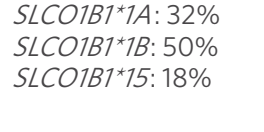 & Prospective & 87 adult patients (Japan) & $\begin{array}{l}\text { MMF } \\
\text { Tacrolimus } \\
\text { Corticosteroids } \\
\text { At day } 28\end{array}$ & $\begin{array}{l}\text { No association of } \\
\text { SLCO1B1* } 1 A,{ }^{*} 1 \mathrm{~B} \text { and } \\
{ }^{*} 15 \text { variants with MPA } \\
\text { pharmacokinetics }\end{array}$ & $\begin{array}{l}\text { No association of } \\
\text { SLCO1B1 }{ }^{*} 1 \mathrm{~A},{ }^{*} 1 \mathrm{~B} \text { and }{ }^{*} 15 \\
\text { variants with diarrhea }\end{array}$ & [65] \\
\hline & $\begin{array}{l}\text { rs } 11045819 \\
(463 C>A)\end{array}$ & $\begin{array}{l}-11187 A: 6 \% \\
\text { c.388A: } 50 \% \\
\text { c.521C: } 14 \%\end{array}$ & $\begin{array}{l}\text { Retrospective (Transgene } \\
\text { study) }\end{array}$ & $\begin{array}{l}218 \text { adult patients } \\
\text { (France) }\end{array}$ & $\begin{array}{l}\text { MMF } \\
\text { Cyclosporine } \\
\text { Tacrolimus } \\
\text { mTOR inhibitor }\end{array}$ & - & $\begin{array}{l}\text { SLCO1B1 c.521C allele } \\
\text { was associated with } \\
\text { reduced MPA-related }\end{array}$ & [13] \\
\hline & $\begin{array}{l}\text { rs } 4149056 \\
\text { (c.521T>C or Val174Ala) }\end{array}$ & & & & $\begin{array}{l}\text { Corticosteroids } \\
\text { One-year follow-up }\end{array}$ & & $\begin{array}{l}\text { cyclosporine-free } \\
\text { treatment }\end{array}$ & \\
\hline & $\begin{array}{l}\text { rs11045819 } \\
\text { (Pro155Thr) }\end{array}$ & $\begin{array}{l}\text { c.388G: } 44 \% \\
\text { 463A: } 14 \% \\
\text { c.521C: } 18 \%\end{array}$ & $\begin{array}{l}\text { Prospective (included } \\
\text { multicenter Apomygre } \\
\text { study) }\end{array}$ & $\begin{array}{l}185 \text { patients } \\
\text { (France) }\end{array}$ & $\begin{array}{l}\text { MMF } \\
\text { Cyclosporine } \\
\text { Tacrolimus } \\
\text { Sirolimus } \\
\text { Corticosteroids } \\
\text { At a point between 3-6 } \\
\text { months }\end{array}$ & $\begin{array}{l}\text { No association of } \\
\text { SLCO1B1 c. } 388 \mathrm{~A}>\mathrm{G}, \\
463 \mathrm{C}>\mathrm{A} \text { and } \mathrm{c} .521 \mathrm{~T}>\mathrm{C} \\
\text { variants with MPA or } \\
\text { MPAG pharmacokinetics }\end{array}$ & - & [14] \\
\hline & & $\begin{array}{l}\text { c.388G: } 46 \% \\
\text { c.521C: } 17 \%\end{array}$ & $\begin{array}{l}\text { Prospective } \\
\text { (FDCC Study) }\end{array}$ & 338 adult patients & $\begin{array}{l}\text { MMF } \\
\text { Cyclosporine } \\
\text { Tacrolimus } \\
\text { Corticosteroids } \\
\text { One-year follow-up }\end{array}$ & $\begin{array}{l}\text { No association of } \\
\text { SLCO1B1 c. } 388 \mathrm{~A}>\mathrm{G} \text { and } \\
\text { c. } 521 T>C \text { variants with } \\
\text { exposure to MPA, MPAG } \\
\text { or AcMPAG }\end{array}$ & $\begin{array}{l}\text { No association of } \\
\text { SLCO1B1 c. } 388 \mathrm{~A}>\mathrm{G} \text { and } \\
\text { c. } 521 \mathrm{~T}>\mathrm{C} \text { variants with } \\
\text { diarrhea or leukopenia }\end{array}$ & [59] \\
\hline & & - & $\begin{array}{l}\text { Retrospective (Case- } \\
\text { Control), multicenter }\end{array}$ & $\begin{array}{l}284 \text { pediatric and young } \\
\text { adult patients (USA) }\end{array}$ & $\begin{array}{l}\text { MMF } \\
\text { Cyclosporine } \\
\text { Tacrolimus } \\
\text { Sirolimus } \\
\text { Corticosteroids } \\
\text { One-year follow-up }\end{array}$ & - & $\begin{array}{l}\text { No association of } \\
\text { SLCO1B1 rs } 4149056 \text { and } \\
\text { rs } 11045819 \text { variants with } \\
\text { leukopenia }\end{array}$ & [27] \\
\hline & & c.521C: $20 \%$ & $\begin{array}{l}\text { Retrospective (Case- } \\
\text { control) }\end{array}$ & $\begin{array}{l}145 \text { adult patients } \\
\text { (Brazil) }\end{array}$ & $\begin{array}{l}\text { MMF } \\
\text { Cyclosporine } \\
\text { Tacrolimus } \\
\text { Sirolimus } \\
\text { Corticosteroids }\end{array}$ & - & $\begin{array}{l}\text { No association of } \\
\text { SLCO1B1 c. } 521 T>C \\
\text { variant with AR }\end{array}$ & [22] \\
\hline & & $\begin{array}{l}\text { c. } 388 \mathrm{~A}: 44 \% \\
\text { c.521C: } 14 \%\end{array}$ & Prospective & 97 adult patients (Brazil) & $\begin{array}{l}\text { EC-MPS } \\
\text { Tacrolimus } \\
\text { Corticosteroids } \\
\text { One-year follow-up }\end{array}$ & $\begin{array}{l}\text { No association of } \\
\text { SLCO1B1 } c .388 \mathrm{~A}>\mathrm{G} \text { and } \\
\text { c. } 521 T>C \text { variants with } \\
\text { MPA C/D }\end{array}$ & $\begin{array}{l}\text { No association of } \\
\text { SLCO1B1 C. } 388 A>G \text { and } \\
\text { C. } 521 T>C \text { variants with } \\
\text { DGF or AR }\end{array}$ & [24] \\
\hline
\end{tabular}




\begin{tabular}{|c|c|c|c|c|c|c|c|c|}
\hline Gene & Variant & Allele frequency & Study design & Population & $\begin{array}{c}\text { Immunosuppressive } \\
\text { regimen }\end{array}$ & Pharmacokinetics & Clinical outcomes & Ref. \\
\hline & & $\begin{array}{l}\text { c.388G: } 48 \% \\
463 \mathrm{~A}: 10 \%\end{array}$ & Prospective & $\begin{array}{l}21 \text { adult patients } \\
\text { (Canada) }\end{array}$ & $\begin{array}{l}\text { MMF } \\
\text { Tacrolimus } \\
\text { Corticosteroid-free } \\
\text { One-year follow-up }\end{array}$ & $\begin{array}{l}\text { No association of } \\
\text { SLCO1B1 c.388A }>G \text { and } \\
463 C>A \text { variants with } \\
\text { MPA exposure (AUC) }\end{array}$ & $\begin{array}{l}\text { No association of } \\
\text { SLCO1B1 c.388A }>\mathrm{G} \text { and } \\
463 C>A \text { variants with } \\
\text { neutrophil counts }\end{array}$ & [49] \\
\hline \multirow[t]{8}{*}{ SLCO1B3 } & $\begin{array}{l}\text { rs4149117 (c.334T>G) } \\
\text { rs7311358 } \\
(c .699 \mathrm{G}>\mathrm{A})\end{array}$ & $\begin{array}{l}\text { c.334T: } 34 \% \\
\text { 699G: } 34 \%\end{array}$ & Prospective & $\begin{array}{l}87 \text { adult patients } \\
\text { (Japan) }\end{array}$ & $\begin{array}{l}\text { MMF } \\
\text { Tacrolimus } \\
\text { Corticosteroids } \\
\text { At day } 28\end{array}$ & $\begin{array}{l}\text { SLCO1B3 c.334GG (699AA) } \\
\text { genotype was associated } \\
\text { with higher exposure to } \\
\left.\text { MPA (AUC }{ }_{6-12}\right)\end{array}$ & $\begin{array}{l}\text { No association of } \\
\text { SLCO1B3 c.334T }>\text { G (or } \\
699 G>A \text { ) variant with } \\
\text { diarrhea }\end{array}$ & [65] \\
\hline & $\begin{array}{l}\text { rs } 1104585 \\
\text { rs60140950 } \\
\text { (c.767G>C) }\end{array}$ & c.334T: $17 \%$ & $\begin{array}{l}\text { Retrospective (Transgene } \\
\text { study) }\end{array}$ & $\begin{array}{l}218 \text { adult patients } \\
\text { (France) }\end{array}$ & $\begin{array}{l}\text { MMF } \\
\text { Cyclosporine } \\
\text { Tacrolimus } \\
\text { mTOR inhibitor } \\
\text { Corticosteroids } \\
\text { One-year follow-up }\end{array}$ & - & $\begin{array}{l}\text { No association of } \\
\text { SLCO1B3 c.334T }>\text { G } \\
\text { variant with AR or } \\
\text { adverse events }\end{array}$ & [13] \\
\hline & & $\begin{array}{l}\text { c.334T: } 18 \% \\
\text { 767C: } 15 \%\end{array}$ & $\begin{array}{l}\text { Prospective (included } \\
\text { multicenter Apomygre } \\
\text { study) }\end{array}$ & $\begin{array}{l}185 \text { patients } \\
\text { (France) }\end{array}$ & $\begin{array}{l}\text { MMF } \\
\text { Cyclosporine } \\
\text { Tacrolimus } \\
\text { Sirolimus } \\
\text { Corticosteroids } \\
\text { At a point between 3-6 } \\
\text { months }\end{array}$ & $\begin{array}{l}\text { SLCO1B3 c. } 334 \mathrm{GG} \\
\text { genotype was associated } \\
\text { with lower MPA peak } \\
\text { concentration and } \\
\text { exposure (AUC } \text { C.12) }_{\text {and }} \\
\text { higher MPAG/MPA ratio, } \\
\text { on cyclosporine-free } \\
\text { treatment }(n=70)\end{array}$ & - & [14] \\
\hline & & c.334T: 19\% & $\begin{array}{l}\text { Prospective (FDCC } \\
\text { Study) }\end{array}$ & 338 adult patients & $\begin{array}{l}\text { MMF } \\
\text { Cyclosporine } \\
\text { Tacrolimus } \\
\text { Corticosteroids } \\
\text { One-year follow-up }\end{array}$ & $\begin{array}{l}\text { No association of } \\
\text { SLCO1B3 c. } 334 T>G \text { (or } \\
699 \mathrm{G}>\mathrm{A} \text { ) variant with } \\
\text { exposure to MPA, MPAG } \\
\text { or AcMPAG }\end{array}$ & $\begin{array}{l}\text { No association of } \\
\text { SLCO1B3 c.334T }>\text { G (or } \\
699 \mathrm{G}>\mathrm{A} \text { ) variant with } \\
\text { diarrhea or leukopenia }\end{array}$ & [59] \\
\hline & & c.334T: $30 \%$ & $\begin{array}{l}\text { Prospective, } \\
\text { Multicenter } \\
\text { (Case-control) }\end{array}$ & $\begin{array}{l}37 \text { adult patients } \\
\text { (China) }\end{array}$ & $\begin{array}{l}\text { MMF } \\
\text { Cyclosporine } \\
\text { Corticosteroids }\end{array}$ & $\begin{array}{l}\text { No association of } \\
\text { SLCO1B3 c. } 334 T>G \\
\text { variant with MPA or } \\
\text { MPAG exposure }\left(A \cup C_{4-12}\right. \\
\left.\text { or } A \cup C_{0-12}\right)\end{array}$ & - & [37] \\
\hline & & c.334T: 15\% & $\begin{array}{l}\text { Prospective, multicenter } \\
\text { (Dominos study) }\end{array}$ & $\begin{array}{l}189 \text { adult patients } \\
\text { (France) }\end{array}$ & $\begin{array}{l}\text { EC-MPS } \\
\text { Cyclosporine } \\
\text { Corticosteroids } \\
\text { Six-month follow-up }\end{array}$ & - & $\begin{array}{l}\text { No association of } \\
\text { SLCO1B3 c. } 334 T>G \\
\text { variant with AR or } \\
\text { adverse events }\end{array}$ & [29] \\
\hline & & - & $\begin{array}{l}\text { Retrospective (Case- } \\
\text { Control), multicenter }\end{array}$ & $\begin{array}{l}284 \text { pediatric and young } \\
\text { adult patients (USA) }\end{array}$ & $\begin{array}{l}\text { MMF } \\
\text { Cyclosporine } \\
\text { Tacrolimus } \\
\text { Sirolimus } \\
\text { Corticosteroids } \\
\text { One-year follow-up }\end{array}$ & - & $\begin{array}{l}\text { No association of } \\
\text { SLCO1B3 c.334T }>G \text { and } \\
\text { rs } 1104585 \text { variants with } \\
\text { leukopenia }\end{array}$ & [27] \\
\hline & & c.334T: $29 \%$ & Retrospective & $\begin{array}{l}408 \text { adult patients } \\
\text { (China) }\end{array}$ & $\begin{array}{l}\text { MMF } \\
\text { Cyclosporine } \\
\text { Tacrolimus } \\
\text { Corticosteroids } \\
\text { 40-day follow-up }\end{array}$ & $\begin{array}{l}\text { No association of } \\
\text { SLCO1B3 c.334T >G } \\
\text { variant with MPA C/D } \\
\text { (days 3-8) }\end{array}$ & - & [38] \\
\hline
\end{tabular}




\begin{tabular}{|c|c|c|c|c|c|c|c|c|}
\hline Gene & Variant & Allele frequency & Study design & Population & $\begin{array}{c}\text { Immunosuppressive } \\
\text { regimen }\end{array}$ & Pharmacokinetics & Clinical outcomes & Ref. \\
\hline \multirow[t]{2}{*}{$S \angle C O 2 B 1$} & $\begin{array}{l}\text { rs } 2306168(S \angle C O 2 B 1 * 3, \\
1457 C>T) \\
\text { rs } 2851069 \\
(c .-71 T>C)\end{array}$ & $S \angle C O 2 B 1 * 3: 30 \%$ & Prospective & 87 adult patients (Japan) & $\begin{array}{l}\text { MMF } \\
\text { Tacrolimus } \\
\text { Corticosteroids } \\
\text { At day } 28\end{array}$ & $\begin{array}{l}\text { No association of } \\
\text { SLCO2B1*3 variant with } \\
\text { MPA pharmacokinetics }\end{array}$ & $\begin{array}{l}\text { No association of } \\
S \angle C O 2 B T^{*} 3 \text { variant with } \\
\text { diarrhea }\end{array}$ & {$[65]$} \\
\hline & & c. $-71 C: 42 \%$ & Prospective & 97 adult patients (Brazil) & $\begin{array}{l}\text { EC-MPS } \\
\text { Tacrolimus } \\
\text { Corticosteroids } \\
\text { One-year follow-up }\end{array}$ & $\begin{array}{l}\text { No association of } \\
\text { SLCO2B1 c. }-71 T>C \\
\text { variant with MPA C/D }\end{array}$ & $\begin{array}{l}\text { No association of } \\
S \angle C O 2 B 1 \text { c.- } 71 T>C \\
\text { variant with DGF or AR }\end{array}$ & {$[24]$} \\
\hline
\end{tabular}

AcMPAG: acyl-MPA-glucuronide; AR: allograft rejection; AUC: area under the concentration-time curve; C/D: trough dose-adjusted concentration; DGF: delayed graft function; EC-MPS: entericcoated mycophenolate sodium salt; eGFR: estimated glomerular filtration rate; MMF: mycophenolate mofetil; MPA: mycophenolic acid; MPAG: MPA-7-O-glucuronide

confirmed by Varnell et al. ${ }^{[27]}$ in a case-control study with children and young adult kidney transplant recipients. On the other hand, CYP2C8 rs11572076GG genotype was associated with reduced risk of leukopenia (GG vs. GA; OR: 0.14, 95\%CI: 0.03-0.59, $P=0.008$ ) with no association with AR, diarrhea or anemia $^{[29]}$. One limiting and common aspect to these studies is the low frequency of the CYP2C8 rs11572076 variant in North American and Canadian (3\%) and French $(0.5 \%)$ patients ${ }^{[28,29]}$. Moreover, there is a minor involvement of CYP2C8 enzyme in MPA metabolism, which makes it difficult to interpret the findings.

In Brazilian patients on EC-MPS and tacrolimus treatment, our group showed that the $C Y P 2 C 8^{*} 3$ (rs11572080 + rs10509681) variant was associated with higher estimated glomerular filtration rate (eGFR), but not with AR, delayed graft function (DGF), or presence of adverse events ${ }^{[24,30]}$. The frequency of the $\mathrm{CYP} 2 \mathrm{C}^{*} 3$ variant was also low $(9 \%)$ in these cohorts.

\section{UGT1A1, UGT1A7, UGT1A8, UGT1A9 and UGT2B7}

Intestinal and hepatic UGTs have important roles in MPA metabolism [Figure 1]. UGT1A9 and UGT1A8 play a main role in MPAG production, and UGT2B7 and UGT1A8 in AcMPAG production, while UGT1A1, UGT1A7 and UGT1A10 plays a minor role in MPA glucuronidation ${ }^{[1,9,31]}$.

The human UGT1 A family (1A1, 1A3-1A10) is encoded by a single locus on chromosome band 2q37. UGT1A1 and UGT1A9 are expressed in the liver and extrahepatic tissues, where UGT1A9 is highly expressed in the kidney and shows lower expression levels in colon, adrenal and bladder ${ }^{[32]}$. UGT1A7, UGT1A8 and UGT1A10 are absent in the liver but are expressed in other tissues, such as the intestine ${ }^{[32]}$. The human UGT2 family is located on chromosome $4 \mathrm{q} 13$. UGT2B7 is expressed in a broad range of human tissues including liver, small intestine, and kidney ${ }^{[32]}$.

UGT1A1 and UGT1A7

Given the known minimal contribution of UGT1A1, UGT1A7 and UGT1A10 to MPA metabolism, there are few pharmacogenetic studies with these isoforms.

Satoh et al. ${ }^{[23]}$ investigated the influence of the $U G T 1 A 1^{*} 28$ (rs3064744) variant in 30 Japanese adult patients. The authors expected to find an association between this UGT1A1 polymorphism and MPA toxicity, but they did not obtain any significant result. 
Four other studies evaluated the impact of UGT1A7 variants in kidney transplant recipients. In three cohorts, UGT1A7 $\operatorname{rs7586110}(-57 \mathrm{~T}>\mathrm{G}), U G T 1 A 7^{*} 2$ and $U G T 1 A 7^{*} 3$ did not influence MPA pharmacokinetics or MMF-related gastrointestinal adverse events or leukopenia ${ }^{[27,33,34]}$. However, UGT1A7 c.622CC (rs11692021) genotype was associated with an accumulation of MPAG (higher $\mathrm{AUC}_{0-12}$ ) without detectable changes in MPA exposure $\left(\mathrm{AUC}_{0-12}\right)$ in Chinese patients ${ }^{[35]}$.

\section{UGT1A8}

The variant $U G T 1 A 8$ rs1042597 (p.Ala173Gly, c.518C > G), also known as $U G T 1 A 8^{\star} 2$, is a missense variant that has a negligible role on protein activity in generating MPAG, but shows decreased production of $A c M P A G^{[31]}$. Consistent with this finding, no association of $U G T 1 A 8^{*} 2$ variant with MPA or MPAG pharmacokinetics was found in kidney transplant recipients ${ }^{[14,36-38]}$. Nevertheless, in agreement with reduced enzyme activity, $U G T 1 A 8^{*} 2$ variant was associated with higher MPA $\mathrm{AUC}_{0-12}$, in cyclosporine-treated patients from the FDCC Study ${ }^{[39]}$, and lower MPAG AUC ${ }_{0-12}$ in Chinese on tacrolimus based treatment ${ }^{[35]}$.

UGT1A8 rs17863762 (p.Cys277Tyr, c.830G>A, UGT1A8*3) is another missense variant, that also induced a reduction in the formation of MPAG in vitro ${ }^{[0,31]}$, and did not impact MPA pharmacokinetics in pediatric or adult patients, probably because of its low frequency in the studied populations (UGT1A8 c.830A: 1\% or $2 \%)^{[39,40]}$.

Regarding the clinical outcomes, Woillard et al. ${ }^{[34]}$ confirmed that MMF treatment combined with tacrolimus and sirolimus increased the risk of diarrhea when compared with cyclosporine. Moreover, only in cyclosporine-treated patients, did the authors demonstrate that $U G T 1 A 8^{*} 2$ variant (c.518G allele) was associated with lower risk of MMF-related diarrhea. The supposed mechanism for this protection involved the decrease in intestinal exposure to $\mathrm{AcMPAG}^{[34]}$. It is known that local (not systemic) exposure to AcMPAG contributes to the toxicity to the intestinal mucosa ${ }^{[3]}$. However, in North American kidney transplant recipients up two weeks, UGT1A8 c.518GG (UGT1A $\left.8^{*}{ }^{*}{ }^{*} 2\right)$ genotype carriers had higher severity of gastrointestinal disorders (abdominal pain, acid reflux, indigestion, diarrhea and constipation) compared to UGT1A8 c.518C allele carriers ${ }^{[41]}$. This finding was not confirmed in final adjusted analysis $(P=0.069)$. Woillard et al ${ }^{[34]}$ found that the results were not similar when they studied the relationship of $U G T 1 A 8^{*} 2$ with diarrhea alone or together with abdominal pain, nausea/vomiting and anorexia, which constituted overly heterogeneous phenotypes.

The occurrence of infections, but not diarrhea or blood disorders, was associated with high dose ( $2 \mathrm{~g} /$ day) MMF treatment and the UGT1 $A^{*} 8^{*_{3}} /{ }^{*} 3(\mathrm{c} .830 \mathrm{AA})$ genotype ${ }^{[42]}$. The authors suggested that this finding was due to increased MPA levels and immunosuppression, in accordance with the lower enzyme activity associated with this variant.

As described in Table 1, UGT1A8 c.518C $>\mathrm{G}$ and c.830G $>$ A variants were not associated with $\mathrm{AR}$, diarrhea, leukopenia or anemia in other investigations with kidney recipients ${ }^{[27,29,39,43]}$.

\section{UGT1A9}

UGT1A9 but not UGT1A8 is expressed in the liver at high levels and is considered the most important hepatic UGT enzyme involved in MPAG formation from MPA ${ }^{[9,32]}$.

The variants UGT1A9 $\mathrm{rs} 6714486$ (c.-275T>A) and $\mathrm{rs} 17868320$ (c.-2152C $>\mathrm{T}$ ) lead to increased protein expression and activity in liver microsomes ${ }^{[44]}$. Accordingly, these polymorphisms were associated with lower MPA exposure and enterohepatic recirculation $\left(\mathrm{AUC}_{6-12}\right.$ used as marker) in kidney patients on MPA and tacrolimus treatment ${ }^{[3,45,46]}$. Kuypers et al. ${ }^{[47]}$ also reported this finding but only among patients treated with $2 \mathrm{~g}$ MMF, and van Schaik et al. ${ }^{[39]}$ reported that the association was dependent on tacrolimus 
treatment. On the contrary, in patients treated with cyclosporine or macrolides, no association with MPA or MPAG pharmacokinetics was found by others ${ }^{[14,40,48,49]}$.

UGT1A9 rs2741046 (c.-331T>C), in complete linkage with rs2741045 (c.-440C>T) ${ }^{[44]}$, rs10176426 $(-665 \mathrm{C}>\mathrm{T})$ and rs13418420 $(-1818 \mathrm{~T}>\mathrm{C})$ variants in the promoter region, also showed conflicting results. In an Italian cohort treated with MMF, cyclosporine and corticosteroid-free regimen, UGT1A9 c.-440 TT and -665CT genotypes were associated with higher MPA exposure $\left(\mathrm{AUC}_{0-2,0-4,0-12}\right.$ or $\left.\mathrm{C} / \mathrm{D}\right)$, but not with MPAG $\mathrm{AUC}$.

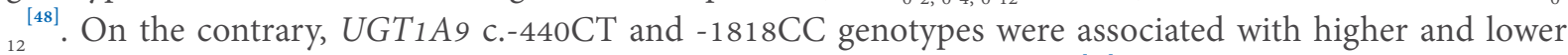
MPAG $\mathrm{AUC}_{0-12}$, respectively, in Chinese patients on tacrolimus treatment ${ }^{[35]}$. Moreover, no association was found with MPA or MPAG pharmacokinetics in other studies ${ }^{[1,38]}$.

The variants UGT1A9 rs3832043 (-118delT), rs72551330 (c.98T>C) and rs2741049 (I399T>C or IVS1 $+399 \mathrm{~T}>\mathrm{C}$ ) do not seem to influence MPA or MPAG pharmacokinetics in kidney transplant patien $\mathrm{ts}^{[14,33,35,38,40,47,49,50]}$. Nevertheless, higher MPA AUC ${ }_{0-12}$ was associated with the presence of UGT1A9 c.98TC genotype in one study with 338 patients ${ }^{[39]}$. Although this result is in accordance with the low activity related to UGT1A9 c.98T $>\mathrm{C}$ variant, the c.98C allele frequency was $2 \%{ }^{[9,39]}$. Furthermore, the low frequency of the UGT1A9 c.-275T>A, c. $-2152 \mathrm{C}>\mathrm{T},-665 \mathrm{C}>\mathrm{T}$ and c.98T $>\mathrm{C}$ polymorphisms was an important limitation of the investigations included in Table 1.

Regarding the clinical outcomes, many investigations showed that the UGT1A9 c.-2152C>T, $-665 \mathrm{C}>\mathrm{T}$, c. $-275 \mathrm{~T}>\mathrm{A}$, c. $-440 \mathrm{C}>\mathrm{T}$, and c. $98 \mathrm{~T}>\mathrm{C}$ variants were not associated with AR and MPA-related adverse events $^{[13,22,27,29,34,47-49]}$.

In line with the aforementioned subtherapeutic levels of immunosuppression, UGT1A9 c.-2152C $>\mathrm{T}$ and c.$275 \mathrm{~T}>\mathrm{A}$ variants were associated with increased risk for AR (OR: 13.3, 95\%CI: $1.1-162.3, P=0.042)^{[39]}$ in patients treated with MPA and tacrolimus. However, another investigation found that UGT1A9 c.-275A (or c. $-2152 \mathrm{~T}$ ) allele carriers had more incidence of gastrointestinal adverse events, which included diarrhea, abdominal pain, reflux, heartburn and constipation, compared to non-carriers ${ }^{[46]}$. A greater severity of diarrhea and heartburn was also associated with these variants. Moreover, the UGT1A9 rs6744284CC genotype was associated with higher severity of constipation (Gastrointestinal Symptom Rating Scale score) at week 1 after transplantation of a North American cohort $^{[41]}$.

In pediatric kidney patients, UGT1 A9 c.-331C allele was associated with leukopenia ${ }^{[43]}$, possibly because of increased systemic MPA exposure ${ }^{[48]}$, which was speculated but not evaluated by the authors.

Pazik et al. ${ }^{[1,52]}$ brought to light the important role of UGT1A9 in reducing exposure to dietary toxins and carcinogens. They showed that UGT1A9 c.98TC genotype was associated with decreased graft function (proteinuria and diminished eGFR), possibly due to reduced detoxifying potential of the UGT1A9 c.98T>C encoded enzyme.

$U G T 2 B 7$

UGT2B7 is the key enzyme involved in AcMPAG formation. Djebli et al. ${ }^{[53]}$ investigated the in vivo and in vitro effect of $U G T 2 B 7$ rs7438135 (c. $-900 \mathrm{G}>\mathrm{A}$, otherwise termed c. $-840 \mathrm{G}>\mathrm{A}$ or c. $-842 \mathrm{G}>\mathrm{A}$ ) and rs7439366 $(802 \mathrm{C}>\mathrm{T})$ variants, which were in complete reverse linkage disequilibrium. The authors found that AcMPAG production was higher in the presence of UGT2B7 c.-900A allele compared to c.-900GG genotype in human liver microsomes. Moreover, the same authors showed that in patients treated with sirolimus $(n=40)$, but not with calcineurin inhibitors (CNIs), UGT2B 7 c.-900AA (or 802CC) genotype was associated with higher AcMPAG $\mathrm{AUC}_{0-9}$ at month 1 and 3 after transplantation ${ }^{[53]}$. Likewise, no association with exposure to AcMPAG was found in other studies with adult patients treated with tacrolimus or 
cyclosporine $e^{[48,54]}$.

The effect of $U G T 2 B 7$ polymorphisms on MPA exposure in kidney recipients also was investigated. UGT2B7 c.-900G $>A$ and $802 \mathrm{C}>\mathrm{T}$ variants had no effect on MPA pharmacokinetics in patients treated with or without corticosteroids, which may induce glucuronidation and result in low MPA exposure ${ }^{[36,39,49,53,55]}$. Baldelli et al ${ }^{[48]}$ found that the UGT2B $7802 \mathrm{TT}$ genotype was associated with higher MPA peak concentration without differences in $\mathrm{AUC}_{0-12}$ MPA. Likewise, UGT2B7-79G>A variant, which is linked to the $802 \mathrm{C}>\mathrm{T}$ and shows a decline in the transcriptional activity of the reporter gene in Caco-2 and HepG2 cells, was not associated with MPA exposure ${ }^{[39,56]}$. On the contrary, MMF apparent oral clearance (CL/ F) was significantly higher in pediatric patients with UGT2B7 $802 \mathrm{~T}$ allele compared to UGT2B7 $802 \mathrm{CC}$ genotype carriers, early after transplantation $(60 \text { days })^{[40]}$. It is important to consider here that differences in metabolism between adults and children may modify the effect of the genetic variants ${ }^{[27,57]}$.

UGT2B7 rs62298861 (IVS1 + 985A>G), rs12233719 (c.211G>T) and rs7662029 (c.-327G>A) variants were also investigated in kidney transplantation. UGT2B7 IVS $1+985 \mathrm{AG}$ and c.211GT genotypes were found to be associated with higher MPA exposure ${ }^{[35,50]}$, but the association with UGT2B7 c.211G>T was not confirmed by another study ${ }^{[49]}$. Li et al ${ }^{[38]}$ found an association between $U G T 2 B 7$ rs7662029 (c.-327G $>A$ ) GG genotype and a higher MPA C/D (days 3-8) in 408 Chinese treated with tacrolimus or cyclosporine, but this result was not confirmed through multiple comparison tests correction.

In a retrospective study of Brazilian adult patients, $U G T 2 B 7$ c.- $900 \mathrm{G}$ allele was associated with prevention of AR (OR: 0.41, 95\%CI: 0.19-0.92, $P=0.030)^{[22]}$. Moreover, the UGT2B7 c.-900G allele was associated with increased risk of leukopenia (OR: 5.3, 95\%CI: 1.1-25.0, $P=0.038)^{[27]}$, only on treatment with non-depleting antibodies, and anemia (GA vs. AA, OR: 1.7, 95\%CI: 1.1-2.4, $P=0.010$; GG vs. AA, OR: 1.9, 95\%CI: 1.2-2.0, $P=0.003)^{[29]}$. All these findings suggest that the UGT2B7 c.-900G allele is associated with higher exposure to MPA, known to favor leukopenia and anemia, and decreased incidence of $\mathrm{AR}^{[3]}$. Woillard et al. ${ }^{[29]}$ also suggested that the relationship between the UGT2B7 c.-900 G>A variant and anemia could be because of the increased production of AcMPAG.

Another investigation failed to find an association of $U G T 2 B 7$ c. $-900 \mathrm{G}>\mathrm{A}, 802 \mathrm{C}>\mathrm{T}$ and $-79 \mathrm{G}>\mathrm{A}$ variants with AR, diarrhea, leukopenia, or other adverse events ${ }^{[13,34,39,43,49,54]}$. Likewise, the variants $r s 12233719$ (c.211G>T) and rs28365063 (c.372A>G) in UGT2B7 were not associated with neutrophil count or graft function in adult patients ${ }^{[30,49]}$.

\section{$A B C B 1, A B C C 2$ and $A B C G 2$}

$\mathrm{ABCB} 1, \mathrm{ABCC} 2$ and $\mathrm{ABCG} 2$ (breast cancer resistance protein, $\mathrm{BCRP}$ ) are expressed in tissues such as intestine, liver and kidney, consistent with their critical role in the absorption, distribution, and elimination of many drugs ${ }^{[58]}$.

Wang et al ${ }^{[18]}$ showed that MPA is a substrate for ABCB1. MPA glucuronide metabolites are substrates for $A B C C 2$, which is considered the main transporter of MPAG from the liver to the biliary system, although this role was suggested also for $\mathrm{ABCG}_{2}{ }^{[15]}$. In the kidneys, $\mathrm{ABCC} 2$ also seems to play an important role in MPAG excretion ${ }^{[19]}$.

$A B C B 1$

$A B C B 1$ is located on chromosome 7, with many variants described so far. Pharmacokinetic studies of MPA have addressed the main investigated polymorphisms of $A B C B 1$ : rs1128503 (c.1236C>T), rs2032582 $(c .2677 \mathrm{G}>\mathrm{T} / \mathrm{A})$, and $\mathrm{rs} 1045642(\mathrm{c} .3435 \mathrm{C}>\mathrm{T})$, which are in linkage disequilibrium ${ }^{[58]}$. 
In studies with kidney recipients, no association of these $\mathrm{ABCB} 1$ variants with exposure to MPA, and MPAG or AcMPAG were observed up to one year after transplantation ${ }^{[23,24,59]}$. Bouamar et al.$^{[59]}$ found that this result was independent of cyclosporine or tacrolimus treatment. It is known that co-administration of cyclosporine leads to lower MPA exposure, by inhibiting enterohepatic recycling of MPA, compared to coadministration of tacrolimus ${ }^{[3]}$. The inhibition of ABCC2 and OATPs by cyclosporine possibly contributes to this drug-drug interaction ${ }^{[18,60]}$. Moreover, cyclosporine is a substrate of $\mathrm{ABCB} 1^{[25]}$.

Satoh et al ${ }^{[23]}$ found that the $A B C B 1 \mathrm{c} .3435 \mathrm{~T}$ allele was associated with greater requirement of MMF dose reduction due to diarrhea in adult patients treated with tacrolimus. Nevertheless, the authors did not find differences in dose-adjusted MPA AUC in ABCB1 c.3435T allele carriers compared to c.3435CC genotype individuals. Thus, they speculated that the interaction of $\mathrm{ABCB} 1$ with other transporters and enzymes might be involved in MPA-induced gastrointestinal toxicity in their small sample size $(n=30)^{[23]} . A B C B 1$ c.3435TT genotype may decrease protein expression or activity, leading to increased drug absorption across the intestine and its higher systemic and intracellular concentrations ${ }^{[61]}$.

On the other hand, in a study with 237 Caucasian patients on MMF and cyclosporine treatment, the $A B C B 1$ c.2677T allele and c.3435T/c.2677T/c.1236T was associated haplotype with increased risk of AR (OR: 3.2, 95\%CI: 1.5-6.7, $P=0.003$ and OR: $2.1,95 \%$ CI: 1.3-3.4, $P=0.002$; respectively), but MPA pharmacokinetic data were unavailable ${ }^{[25]}$. Since low MPA AUC ${ }_{0-12}$ was associated with increased incidence of biopsy-proven acute rejection ${ }^{[3]}$, this result from Grinyó et al ${ }^{[25]}$ collaborates the complex mechanisms of clinical outcomes in immunosuppressive therapy.

Other investigations found no association of $A B C B 1$ c.1236C > T, c.2677G $>\mathrm{T} / \mathrm{A}$, and c.3435C $>\mathrm{T}$ variants with AR, DGF, diarrhea, leukopenia or other MPA-related adverse events in pediatric or adult kidney recipients $^{[13,24,26,27,59]}$.

\section{$A B C C 2$}

The extensively studied variant of $A B C C 2$ (on chromosome 10), the rs717620 (c.-24C>T), is located in the $5^{\prime}$-UTR. The effect of this polymorphism in several in vivo studies is contradictory, suggesting that it is highly tissue specific and dependent on regulatory factors, such as the epigenetics (miRNA expression) ${ }^{[58]}$. Naesens et al. ${ }^{[62]}$ investigated the $A B C C 2$ c. $-24 \mathrm{C}>\mathrm{T}$ variant, in linkage disequilibrium with rs3740066 (c.3972C>T), in adult kidney transplant recipients treated with MMF and tacrolimus for one year. At day 7 , only non-carriers of the $A B C C 2$ c.-24C>T variant and patients with mild liver dysfunction had significantly lower MPA exposure than those without liver disease. This difference was not observed in $A B C C 2$ c.$24 \mathrm{~T}$ allele carriers. The reasons were not clarified. In the same study, from day 42 post-transplantation, the $A B C C 2$ c. $-24 \mathrm{~T}$ allele was associated with higher MPA C/D and AUC ${ }^{[62]}$. The authors suggested that the variant was associated with increased protein expression and/or activity and enhanced enterohepatic recirculation. In line with this, the $A B C C 2$ c.-24TT genotype was also associated with higher MPA C/D (days 3-8) in 408 Chinese patients on cyclosporine or tacrolimus treatment, but this result was not confirmed after Bonferroni correction in the multiple comparisons analysis ${ }^{[38]}$.

van Schaik et al. ${ }^{[39]}$ found that MPA AUC ${ }_{0-12}$ was $17 \%-23 \%$ higher for ABCC2 c.-24C>T carriers, but this difference reached statistical significance only at 6 weeks post-transplantation. Conversely, the $A B C C 2$ c. $-24 \mathrm{~T}$ allele was associated with a lower MPA AUC at month 1, in a study with 55 kidney transplant recipients on tacrolimus and sirolimus therapy ${ }^{[63]}$.

Božina et al ${ }^{[64]}$ did not find any association between the ABCC2 c.-24T variant and MPA pharmacokinetics in kidney recipient or donors, but the A allele of the ABCC2 rs2273697 (Val417lle, c.1249G>A) in kidney donors was associated with a reduced peak $(29 \%)$ and early $\left(\mathrm{AUC}_{0-2}, 33 \%\right)$ exposure to MPA, suggesting 
an increased renal elimination. Moreover, the authors showed that the combination of cyclosporine and $A B C C 2$ c.1249A allele reduced MPA $\mathrm{AUC}_{0-2}$ by $49 \%$, an additive effect of this immunosuppressant.

Lack of association of $A B C C 2$ c. $-24 \mathrm{C}>\mathrm{T}, 1249 \mathrm{G}>\mathrm{A}, \mathrm{c} .3972 \mathrm{C}>\mathrm{T}$, and $\mathrm{rs} 8187694$ (3563T $>\mathrm{A}$ ) variants with MPA or MPAG pharmacokinetics was reported by other studies ${ }^{[14,24,37,39,40,45,48,49,65,66]}$.

Regarding clinical outcomes, Naesens et al. ${ }^{[62]}$ found that $A B C C 2$ c. $-24 \mathrm{~T}$ allele carriers had more frequent episodes of diarrhea that non-carriers in the first year after transplantation. The authors considered the limitation of the sample size but stated that an increased enterohepatic recirculation of MPAG associated with the $A B C C 2$ c. $-24 \mathrm{C}>\mathrm{T}$ variant could lead to diarrhea. It is important to mention that the local exposure and not the systemic concentration of MPA is relevant for gastrointestinal adverse events. Moreover, AcMPAG, produced in gastrointestinal cells and also substrate of ABCC2, contributes to cell toxicity ${ }^{[3,15]}$.

Other studies failed to confirm associations between $A B C C 2$ polymorphisms and clinical outcomes (AR, DGF, graft function) or MPA-related adverse events in pediatric or adult kidney recipien ts $^{[13,22,24,25,27,29,30,34,39,49,65]}$.

\section{ABCG2}

Brazilian, Chinese, and North American cohorts have been studied regarding two ABCG2 variants in pediatric and adult kidney transplant patients [Table 2]. The ABCG2 c.421C>A (rs2231142) variant leads to the amino acid exchange Gln141Lys, whereas ABCG2-20 + 11790G>A (rs4491984) is an intronic polymorphism.

The investigations pointed out that these variants did not influence MPA or MPAG exposure or clinical outcomes during treatment with different immunosuppressive therapies ${ }^{[24,27,30,37,38]}$.

\section{SLC01B1, SLCO1B3 and SLCO2B1}

OATPs additionally contribute to drug disposition, with ABC proteins; however, OATPs do not depend directly on using cellular $\mathrm{ATP}^{[67,68]}$. OATP $1 \mathrm{~B}_{1}$ and $\mathrm{OATP}_{1} \mathrm{~B}_{3}$ are mainly expressed in the liver, while OATP1A2 is expressed in the intestine, biliary cells of the liver, and distal nephron of the kidney ${ }^{[67]}$.

In studies with OATP-transfected human embryonic kidney (HEK) cells, MPAG uptake, but not MPA, was enhanced by OATP1B3 (SLCO1B3) and to a lesser extent by OATP1B1 (SLCO1B1). MPA or MPAG uptake was not influenced by OATP1A2 $(S L C O 1 A 2)^{[14]}$. In line with this, Michelon et al ${ }^{[13]}$ found that MPAG and AcMPAG, but not MPA, were substrates of OATP1B1.

\section{SLCO1B1}

A large number of $S L C O 1 B 1$ variants have been described. The two SLCO1B1 common variants rs2306283 (p.Asn130Asp, c.388A>G) and rs4149056 (p.Val174Ala, c.521T>C) form together four distinct haplotypes: SLCO $1 B 1^{*} 1 A$ (c.388A-c.521T, reference haplotype), ${ }^{*} 1 B($ c. $388 \mathrm{G}-\mathrm{c} .521 \mathrm{~T}),{ }^{{ }^{*}} 5$ (c.388A-c.521C) and ${ }^{*} 15$ (c.388G-c.521C) $)^{[69]}$.

Kidney transplantation with macrolide or cyclosporine-based treatment has shown no association between the SLCO1B1 c.388A>G, c.521T>C, and rs1 1045819 (463C>A) variants or haplotypes with MPA, MPAG or AcMPAG pharmacokinetics ${ }^{[14,24,49,59,65]}$.

Likewise, SLCO1B1 variants have not been associated with AR, DGF, diarrhea, leukopenia or neutrophil count in the studies summarized in Table $2^{[22,24,27,49,59,65]}$. Nevertheless, SLCO1B1 C.521C allele (SLCO1B1 $\left.{ }^{*} 5\right)$ was associated with reduced MPA-related adverse events, including leukopenia, anemia, thrombocytopenia, diarrhea, nausea, vomiting or infection in French patients on cyclosporine-free treatment ${ }^{[13]}$. Possibly 
because cyclosporine inhibits OATP1B1, the effect of the $S L C O 1 B 1$ c.521C allele was not detectable in the therapy with this immunosuppressant ${ }^{[13,60]}$. Indeed, cyclosporine-based treatment and SLCO1B1 C.521C allele were independent factors related to risk reduction of adverse events (OR: 0.22, 95\%CI: 0.08-0.56, $P$ $=0.002$ and OR: $0.38,95 \%$ CI: $0.21-0.69, P=0.001$; respectively $)^{[13]}$. It was also demonstrated in vitro the reduced uptake of MPAG and AcMPAG related to SLCO1B1 c.521C variant. The authors hypothesized that carriers of the SLCO1B1 c.521C allele had impaired hepatic uptake of MPAG and AcMPAG, which leads to less enterohepatic recycling and, consequently, reduced exposure to MPA and less adverse events ${ }^{[13]}$. However, this study did not evaluate MPA exposure, and other investigations did not find an association between the SLCO1B1 C.521T>C variant and MPA pharmacokinetics, as previously mentioned.

\section{SLCO1B3}

Four variants in $S L C O 1 B 3$ were investigated in pediatric and adult recipients of kidney transplantation [Table 2]. Three are missense variants, SLCO1B3 rs4149117 (Ser112Ala, c.334T>G), rs7311358 (Met233Ile, c.699G $>$ A) and rs60140950 (Gly256Ala, c.767G>C).

SLCO1B3 c.334T>G and c.699G $>$ A are in linkage disequilibrium, and the SLCO1B3 c.334G-c.699A haplotype was associated with reduced MPAG uptake in HEK293 cells in vitro ${ }^{[14]}$. In a study with 70 patients on tacrolimus and sirolimus treatment, but not on cyclosporine, the SLCO1B3 c.334GG genotype was associated with lower MPA peak concentration and exposure $\left(\mathrm{AUC}_{0-12}\right)$ and higher MPAG/MPA ratio ${ }^{[14]}$. On the basis of in vitro and in vivo results, the authors suggested that reduced OATP1B3 activity would decrease hepatic uptake of MPAG, reducing reabsorption of MPA through enterohepatic cycling in SLCO1B3 c.334GG genotype carriers.

Other investigations did not find an association between $S L C O 1 B 3$ c.334T $>$ G (or c.699G $>$ A) variant and exposure to MPA, MPAG, or AcMPAG during treatment with either cyclosporine, in line with the previous findings ${ }^{[14]}$, or tacrolimus ${ }^{[37,38,59]}$. On the contrary, the SLCO1B3 c.334GG (699AA) genotype was associated with higher MPA $\mathrm{AUC}_{6-12}$, considered a marker of MPA recirculation, in Japanese patients with tacrolimusbased therapy at day 28 after transplantation ${ }^{[65]}$.

SLCO1B3 c.334T $>\mathrm{G}$ (or $699 \mathrm{G}>\mathrm{A}$ ) and $\mathrm{rs1} 104585$ variants were not associated with AR, leukopenia, diarrhea or other adverse events ${ }^{[13,27,29,59,65]}$.

\section{SLCO2B1}

In Brazilian or Japanese kidney recipients, early or long after transplantation, SLCO2B1 c. $-71 \mathrm{~T}>\mathrm{C}$ (rs2851069) and $\mathrm{SLCO}_{2} \mathrm{~B}_{1}{ }_{3}$ (rs2306168) variants did not influence MPA pharmacokinetics or clinical outcomes of tacrolimus based-therapy ${ }^{[24,65]}$.

\section{GENES RELATED TO MPA PHARMACODYNAMICS}

Polymorphisms in IMPDH1 or IMPDH2 that result in increased IMPDH activity are likely to enhance T and $\mathrm{B}$ cell proliferation and decrease the response to MPA in kidney transplantation ${ }^{[1,2]}$. As a result, less sensitivity to MPA requires an increase in the dose of MMF to avoid the risk of acute rejection, but this clinical approach exposes patients to higher blood concentrations of MPA and increases the likelihood of adverse events ${ }^{[1]}$.

The pharmacogenomics studies involving genes related to MPA pharmacodynamics are summarized in the Table 3.

\section{IMPDH1}

A large-scale genomic study investigated variants in $I M P D H 1$ using a gene resequencing approach. It identified 73 variants ( 59 novel) in a cohort of 288 healthy subjects, including four missense variants: 
rs72624960 (Ser275Leu), rs72624961 (Ala285Th), rs61751223 (His296Arg), and rs72624967 (Arg412Trp). Functional analysis revealed that IMPDH1 Leu275 significantly reduced enzyme activity, where structural analysis predicted that the amino acid substitution alters the structure and function of the enzyme ${ }^{[70]}$. The authors also identified two variants previously described in $I M P D H 1$ : the synonymous variant rs2228075 (Ala525Ala, c.1320G>A, C>T) and the intronic variants rs2278293 (c.579 + 119G $>$ A, C $>\mathrm{T}$ ) and rs2278294 (c.580-106G>A, C>T).

The IMPDH1 rs2278293 and rs2278294 (intronic) and rs2228075 (synonymous) variants were investigated in kidney recipients from several populations treated with MMF [Table 3].

Wang et al. ${ }^{[71]}$ examined the contribution of 17 variants in IMPDH1, including rs2228075, rs2278293 and rs2278294, to acute rejection or toxicity in 191 adult kidney recipients from the US on MMF therapy. The rs2278293 A allele (OR: 0.34, 95\%CI:0.15-0.76, $P=0.008$ ) and the rs2278294 A allele (OR: $0.40,95 \% \mathrm{CI}$ : 0.18-0.89, $P=0.02$ ) were associated with reduced risk for acute rejection (AR) in the first postoperative year ${ }^{[71]}$. Gensburger et al. ${ }^{[72]}$ also explored the influence of both IMPDH1 intronic polymorphisms on clinical outcomes, in 456 kidney recipients from the Apomygree and FDCC studies, and found an association of the rs2278294 A allele with reduced AR risk (OR: 0.54, 95\%CI: 0.34-0.85, $P=0.0075$ ), suggesting a protective effect of this variant.

Other studies reported a lack of association of both IMPDH1 rs2278293 and rs2278294 variants with AR in adult patients from the Transgene Study ${ }^{[13]}$ and the Collaborative Transplant Study (the largest cohort, over 1000 kidney recipients) on MMF treatment ${ }^{[73]}$. Similar results were also found in adult kidney recipients treated with EC-MPS from the multicenter Dominos study ${ }^{[29]}$. The IMPDH1 rs2278293 was also not associated with AR in a kidney transplantation study from Brazil ${ }^{[22]}$.

Shah et al. ${ }^{[73]}$ reported the lack of an association of the variants rs2278293 and rs2278294 in IMPDH1 with MMF long-term dose tolerated and dose achieved in adult kidney recipients from the Collaborative Transplant Study. A short-term follow-up study also reported no direct association of both IMPDH1 rs2278294 and rs2278293 polymorphisms with subclinical AR in 82 Japanese adult kidney recipients. However, the interaction of the rs2278293 A allele with high MPA night-time exposure range (AUC > 60 $\mu \mathrm{g} . \mathrm{h} / \mathrm{mL}$ and $\mathrm{C}_{0} \geq 1.9 \mu \mathrm{g} / \mathrm{mL}$ ) increased the risk of subclinical $\mathrm{AR}^{[74]}$.

Two studies investigated the missense variant IMPDH1 rs2228075 (Ala525Ala, c.1320G>A) and the risk for AR, though no significant association was found in MMF-treated adult kidney recipients from the US ${ }^{[71]}$ and from the CAESAR Study ${ }^{[25]}$

The Collaborative Transplant Study explored the variants IMPDH1 rs2278293 and rs2278294 in a large cohort of kidney recipients, and found no association with long-term graft function (one year) and graft survival (five years) ${ }^{[73]}$.

The influence of IMPDH1 variants on hematological and gastrointestinal adverse events related to MMF therapy was also explored in several studies. The IMPDH1 rs2278294 G>A was reported to be associated with increased risk of leukopenia (A allele: OR: 1.66, 95\%CI: 1.11-2.48, $P=0.0139$ ) in adult kidney recipients from the Apomygree and FDCC studies ${ }^{[72]}$. Moreover IMPDH1 rs2278294 G and rs2228075 G alleles were associated with delayed time to leukopenia in children and young adult patients from USA ${ }^{[27]}$. Although these intronic variants were predicted not to affect IMPDH1 function, the authors suggested that these alleles may affect the sensitivity of the enzyme to MPA.

Other studies reported lack of association of IMPDH1 rs2278293, rs2278294 or rs2228075 with leukopenia in adult kidney recipients on MMF therapy from the $\mathrm{US}^{[71]}$, Apomygre and FDCC studies ${ }^{[72]}$, and Transgene 
Table 3. Genes related to mycophenolic acid pharmacodynamics in kidney transplantation

\begin{tabular}{|c|c|c|c|c|c|c|c|}
\hline Gene & Variant & Allele frequency & Study design & Population & $\begin{array}{c}\text { Immunosuppressive } \\
\text { regimen }\end{array}$ & Clinical outcomes & Ref. \\
\hline \multirow[t]{8}{*}{ IMPDH1 } & $\begin{array}{l}\text { rs2228075 C>T } \\
\text { (Ala525Ala, } \\
\text { c.1320G }>\text { A) }\end{array}$ & c.1320A: $22 \%$ & $\begin{array}{l}\text { Prospective, multicenter } \\
\text { (CAESAR study) }\end{array}$ & 237 adult patients & $\begin{array}{l}\text { MMF } \\
\text { Cyclosporine } \\
\text { Corticosteroids } \\
\text { One-year follow-up }\end{array}$ & $\begin{array}{l}\text { No association of /MPDH1 rs2228075 } \\
\text { variant with AR }\end{array}$ & [25] \\
\hline & $\begin{array}{l}\text { rs } 2278293 C>T \\
(c .579+119 G>A) \\
\text { rs2278294 C>T } \\
(c .580-106 G>A)\end{array}$ & $\begin{array}{l}\text { rs2278293 A: } 46 \% \\
\text { rs2278294 A: } 36 \%\end{array}$ & $\begin{array}{l}\text { Prospective, multicenter } \\
\text { (Apomygre and FDCC } \\
\text { studies) }\end{array}$ & 456 adult patients & $\begin{array}{l}\text { MMF } \\
\text { Cyclosporine } \\
\text { Tacrolimus } \\
\text { Corticosteroids } \\
\text { One-year follow-up }\end{array}$ & $\begin{array}{l}\text { IMPDH1 rs2278294 A allele was } \\
\text { associated with reduced risk of AR and } \\
\text { increased risk of leukopenia } \\
\text { No association of rs2278293 with AR, } \\
\text { leukopenia, CMV and other infections }\end{array}$ & {$[72]$} \\
\hline & & $\begin{array}{l}\text { rs2278293 A: } 44 \% \\
\text { rs2278294 A: } 45 \%\end{array}$ & Prospective & 82 adult patients (Japan) & $\begin{array}{l}\text { MMF } \\
\text { Tacrolimus } \\
\text { Corticosteroids } \\
\text { At day } 28\end{array}$ & $\begin{array}{l}\text { No direct association of IMPDH1 } \\
\text { variants with subclinical AR. } \\
\text { IMPDH1 rs2278293 A allele combined } \\
\text { with high MPA night-time exposure } \\
\text { range }\left(A \cup C>60 \mu \text { g.h } / \mathrm{mL} \text { and } C_{0}\right. \\
\cup 1.9 \mu \mathrm{g} / \mathrm{mL}) \text { was associated with } \\
\text { subclinical AR }\end{array}$ & {$[74]$} \\
\hline & & $\begin{array}{l}\text { rs2278293 A: } 49 \% \\
\text { rs2278294 A: } 38 \%\end{array}$ & $\begin{array}{l}\text { Retrospective (Transgene } \\
\text { study) }\end{array}$ & 218 adult patients (France) & $\begin{array}{l}\text { MMF } \\
\text { Cyclosporine } \\
\text { Tacrolimus } \\
\text { mTOR inhibitor } \\
\text { Corticosteroids } \\
\text { One-year follow-up }\end{array}$ & $\begin{array}{l}\text { No association of IMPDH1 variants } \\
\text { with MPA-related AR or leukopenia }\end{array}$ & [13] \\
\hline & & $\begin{array}{l}\text { rs2228075 T: } 25 \% \\
\text { rs2278293 A: } 43 \% \\
\text { rs2278294 A: } 40 \%\end{array}$ & Retrospective & 191 adult patients (USA) & $\begin{array}{l}\text { MMF } \\
\text { Tacrolimus } \\
\text { Prednisone } \\
\text { One-year follow-up }\end{array}$ & $\begin{array}{l}\text { The variants } / M P D H 1 \text { rs } 2278293 \\
\text { and rs } 2278294 \text { were associated with } \\
\text { reduced risk of AR } \\
\text { No association of IMPDH1 variants } \\
\text { with leukopenia }\end{array}$ & [71] \\
\hline & & $\begin{array}{l}\text { rs2278293 T: } 45 \% \\
\text { rs2278294 A: } 40 \%\end{array}$ & $\begin{array}{l}\text { Prospective, multicenter } \\
\text { (Collaborative Transplant } \\
\text { study) }\end{array}$ & 1,040 adult patients & $\begin{array}{l}\text { MMF } \\
\text { Cyclosporine } \\
\text { Tacrolimus } \\
\text { Corticosteroids } \\
\text { One-year follow-up }\end{array}$ & $\begin{array}{l}\text { No association of } I M P D H 1 \text { variants } \\
\text { with MMF doses tolerated ( } 1 \text { year) and } \\
\text { dose achieved ( } 3 \text { years) } \\
\text { No association of IMPDH1 variants } \\
\text { with AR, graft function ( } 1 \text { year) or graft } \\
\text { survival ( } 5 \text { years) }\end{array}$ & {$[73]$} \\
\hline & & $\begin{array}{l}\text { rs22278923 T: } 47 \% \\
\text { rs2278924 T: } 33 \%\end{array}$ & $\begin{array}{l}\text { Prospective, multicenter } \\
\text { (Dominos study) }\end{array}$ & 189 adult patients & $\begin{array}{l}\text { EC-MPS, Cyclosporine } \\
\text { Corticosteroids } \\
\text { Six-month follow-up }\end{array}$ & $\begin{array}{l}\text { No association of } I M P D H 1 \text { variants } \\
\text { with } A R \text {, leukopenia anemia or diarrhea }\end{array}$ & [29] \\
\hline & & $\begin{array}{l}\text { rs2228075: - } \\
\text { rs2278293: - } \\
\text { rs2278294: - }\end{array}$ & $\begin{array}{l}\text { Retrospective (Case- } \\
\text { control), multicenter }\end{array}$ & $\begin{array}{l}284 \text { pediatric and young } \\
\text { adult patients (USA) }\end{array}$ & $\begin{array}{l}\text { MMF } \\
\text { Tacrolimus } \\
\text { Cyclosporine } \\
\text { Sirolimus } \\
\text { Corticosteroids } \\
\text { One-year follow-up }\end{array}$ & $\begin{array}{l}\text { IMPDH1 rs } 2228075 \mathrm{G} \text { and rs } 2278294 \\
\mathrm{G} \text { alleles were associated with } \\
\text { increased time to leukopenia }\end{array}$ & [27] \\
\hline
\end{tabular}




\begin{tabular}{|c|c|c|c|c|c|c|c|}
\hline Gene & Variant & Allele frequency & Study design & Population & $\begin{array}{c}\text { Immunosuppressive } \\
\text { regimen }\end{array}$ & Clinical outcomes & Ref. \\
\hline & & rs2278293 A: 47\% & $\begin{array}{l}\text { Retrospective } \\
\text { (Case-control) }\end{array}$ & 145 adult patients (Brazil) & $\begin{array}{l}\text { MMF } \\
\text { Cyclosporine Tacrolimus } \\
\text { Sirolimus } \\
\text { Corticosteroids } \\
\text { One-year follow-up }\end{array}$ & $\begin{array}{l}\text { No association of IMPDH7 variant with } \\
A R\end{array}$ & {$[22]$} \\
\hline & & $\begin{array}{l}\text { rs2278293 A: } 45 \% \\
\text { rs2278294 A: } 30 \%\end{array}$ & Prospective & $\begin{array}{l}190 \text { adult patients } \\
\text { (Poland) }\end{array}$ & $\begin{array}{l}\text { MMF } \\
\text { Cyclosporine } \\
\text { Tacrolimus } \\
\text { Corticosteroids } \\
\text { Five-year follow-up }\end{array}$ & $\begin{array}{l}\text { IMPDH1 rs2278294 G allele was } \\
\text { associated with slower BMI gain }\end{array}$ & [75] \\
\hline \multirow[t]{7}{*}{ IMPDH2 } & $\begin{array}{l}\text { rs } 11706052 \mathrm{~A}>\mathrm{G} \\
(\mathrm{c} .819+10 \mathrm{~T}>\mathrm{C} \text {, also } \\
\text { known as } 3757 \mathrm{~T}>\mathrm{C})\end{array}$ & 3757C: $7 \%$ & $\begin{array}{l}\text { Prospective, multicenter } \\
\text { (CAESAR study) }\end{array}$ & 237 adult patients & $\begin{array}{l}\text { MMF } \\
\text { Cyclosporine } \\
\text { Corticosteroids } \\
\text { One-year follow-up }\end{array}$ & $\begin{array}{l}\text { IMPDH2 } 3757 \mathrm{C} \text { allele was associated } \\
\text { with increased risk of AR }\end{array}$ & [25] \\
\hline & $\begin{array}{l}\text { rs121434586 } \\
\text { (Leu236Phe, } \\
\text { c.787C>T) }\end{array}$ & $\begin{array}{l}\text { 3757TC+CC: } 9 \% \\
\text { c. } 787 T: 0.3 \%\end{array}$ & Retrospective & 191 adult patients (USA) & $\begin{array}{l}\text { MMF } \\
\text { Tacrolimus } \\
\text { Prednisone } \\
\text { One-year follow-up }\end{array}$ & $\begin{array}{l}\text { No association of } I M P D H 2 \text { variants } \\
\text { with AR or leukopenia }\end{array}$ & [71] \\
\hline & $\begin{array}{l}\text { rs 4974081 } \\
(-3624 A>G)\end{array}$ & 3757C: $7 \%$ & Prospective & $\begin{array}{l}101 \text { adult patients } \\
\text { (Netherlands) }\end{array}$ & $\begin{array}{l}\text { MMF } \\
\text { Tacrolimus } \\
\text { Corticosteroids } \\
\text { One-year follow-up }\end{array}$ & $\begin{array}{l}\text { IMPDH2 3757C allele was associated } \\
\text { with increased MPA plasma } \\
\text { concentration ( } 6 \mathrm{~h} \text { after MMF intake) } \\
\text { and increased IMPDH activity in PBMC } \\
\text { (12 h after MMF intake) } \\
\text { No association of IMPDH2 variant with } \\
\text { AR }\end{array}$ & [78] \\
\hline & & $\begin{array}{l}\text { rs11706052 C: } 9 \% \\
\text { rs4974081 G: } 24 \%\end{array}$ & $\begin{array}{l}\text { Prospective (Apomygre and } \\
\text { FDCC studies) }\end{array}$ & 456 adult patients & $\begin{array}{l}\text { MMF } \\
\text { Cyclosporine } \\
\text { Tacrolimus } \\
\text { Corticosteroids } \\
\text { One-year follow-up }\end{array}$ & $\begin{array}{l}\text { No association of the IMPDH2 } \\
\text { rs } 11706052 \text {, rs } 4974081 \text { variants with } \\
\text { AR, leukopenia or CMV infection }\end{array}$ & {$[72]$} \\
\hline & & 3757C: $8 \%$ & Prospective & $\begin{array}{l}177 \text { adult patients } \\
\text { (Poland) }\end{array}$ & $\begin{array}{l}\text { MMF } \\
\text { Calcineurin inhibitors } \\
\text { Corticosteroids } \\
\text { One-year follow-up }\end{array}$ & $\begin{array}{l}\text { IMPDH2 } 3757 \text { C allele was associated } \\
\text { with increased lymphocyte counts and } \\
\text { reduced risk of lymphopenia } \\
\text { No association of IMPDH2 variant } \\
\text { with AR, neutropenia, gastrointestinal } \\
\text { adverse events or serious infections }\end{array}$ & {$[80]$} \\
\hline & & rs11706052 G: 19\% & $\begin{array}{l}\text { Prospective, multicenter } \\
\text { (Collaborative Transplant } \\
\text { study) }\end{array}$ & 1,040 adult patients & $\begin{array}{l}\text { MMF } \\
\text { Cyclosporine } \\
\text { Tacrolimus } \\
\text { Corticosteroids } \\
\text { One-year follow-up }\end{array}$ & $\begin{array}{l}\text { No association of IMPDH2 variant with } \\
\text { MMF doses tolerated (1-year) and } \\
\text { dose achieved ( } 3 \text {-years) } \\
\text { No association of IMPDH2 variant with } \\
\text { AR, graft function (1 year) or survival } \\
\text { ( } 5 \text { years) }\end{array}$ & [73] \\
\hline & & rs11706052 C: 11\% & $\begin{array}{l}\text { Prospective, multicenter } \\
\text { (Dominos study) }\end{array}$ & 189 adult patients & $\begin{array}{l}\text { EC-MPS } \\
\text { Cyclosporine } \\
\text { Corticosteroids } \\
\text { Six-month follow-up }\end{array}$ & $\begin{array}{l}\text { No association of } / M P D H 2 \text { variant with } \\
A R \text {, leukopenia, anemia or diarrhea }\end{array}$ & [29] \\
\hline
\end{tabular}




\begin{tabular}{|c|c|c|c|c|c|c|c|}
\hline Gene & Variant & Allele frequency & Study design & Population & $\begin{array}{l}\text { Immunosuppressive } \\
\text { regimen }\end{array}$ & Clinical outcomes & Ref. \\
\hline & & $\begin{array}{l}\text { rs11706052 C: - } \\
\text { rs4974081 G: - }\end{array}$ & $\begin{array}{l}\text { Retrospective (Case- } \\
\text { control), multicenter }\end{array}$ & $\begin{array}{l}284 \text { pediatric and young } \\
\text { adult patients (USA) }\end{array}$ & $\begin{array}{l}\text { MMF } \\
\text { Tacrolimus } \\
\text { Cyclosporine } \\
\text { Sirolimus } \\
\text { Corticosteroids } \\
\text { One-year follow-up }\end{array}$ & $\begin{array}{l}\text { No association of } I M P D H 2 \text { variants } \\
\text { with leukopenia or time to leukopenia }\end{array}$ & [27] \\
\hline & & rs11706052 C: 11\% & $\begin{array}{l}\text { Retrospective } \\
\text { (Case-control) }\end{array}$ & 145 adult patients (Brazil) & $\begin{array}{l}\text { MMF } \\
\text { Cyclosporine Tacrolimus } \\
\text { Sirolimus } \\
\text { Corticosteroids } \\
\text { One-year follow-up }\end{array}$ & $\begin{array}{l}\text { IMPDH2 rs11706052 C allele was } \\
\text { associated with reduced risk of AR }\end{array}$ & [22] \\
\hline & & rs11706052 C: 8\% & Prospective & $\begin{array}{l}190 \text { adult patients } \\
\text { (Poland) }\end{array}$ & $\begin{array}{l}\text { MMF } \\
\text { Cyclosporine } \\
\text { Tacrolimus } \\
\text { Corticosteroids } \\
\text { Five-year follow-up }\end{array}$ & $\begin{array}{l}\text { No association of IMPDH2 variant with } \\
\text { BMI change }\end{array}$ & {$[75]$} \\
\hline
\end{tabular}

AR: acute rejection; AUC: area under the concentration-time curve; BMI: body mass index; CMV cytomegalovirus; EC-MPS: enteric-coated mycophenolate sodium; MMF: mycophenolate mofetil; MPA: mycophenolyc acid

Study ${ }^{[13]}$. Likewise both rs2278294 and rs2278293 polymorphisms were not associated with leukopenia or anemia in adult patients from the Dominos Study within six months of EC-MPS treatment ${ }^{[29]}$. Eight other variants in IMPDH1 were also identified by Wang et al. ${ }^{[71]}$, but no association with leukopenia was found.

Woillard et al. ${ }^{[29]}$ also investigated the influence of IMPDH1 rs2278923 and rs2278924 polymorphisms on gastrointestinal adverse events, such as diarrhea, but no association was found in adult kidney recipients from Dominos Study on EC-MPS therapy. Likewise, no association was found between these variants and susceptibility to CMV and other infections in adult kidney recipients on MMF therapy from the Apomygre and FDCC studies ${ }^{[72]}$.

Pazik et al. ${ }^{[75]}$ explored the influence of IMPDH1 rs2278923 and rs2278924 on time-dependent change in body mass index (BMI) of adult kidney recipients from Poland, and found an association of the rs2278294 G allele with slower BMI gain over five years post-transplantation.

\section{IMPDH2}

Wu et al. ${ }^{[70]}$ identified 25 variants (24 novel) in $\mathrm{IMPDH} 2$, including the missense rs72624919 (Ser485Cys), in 288 healthy subjects. The functional analysis was carried out with Ser485Cys and Leu263Phe (rs121434586, c.787C > T), a missense variant previously described ${ }^{[76]}$. The IMPDH2 Phe263, but not the Cys485, reduced markedly protein level and enzymatic activity, and altered the structure and function of the enzyme. They also detected the intronic variant $I M P D H 2$ rs72639214 (IVS1-93), which was associated with reduced IMPDH2 basal mRNA expression in lymphoblastoid cell lines, as well as the intronic variant $I M P D H 2$ rs11706052 $\mathrm{A}>\mathrm{G}(\mathrm{c} .819+10 \mathrm{~T}>\mathrm{C})$ also known as $3757 \mathrm{~T}>\mathrm{C}^{[70]}$. 
Ten studies explored the IMPDH2 rs121434586 (Leu263Phe), rs1 1706052 (intronic) and rs4974081 (-3624 $A>G)$, a 5 'upstream variant which was predicted to be a potential transcription factor binding site ${ }^{[77]}$, in MMF-treated kidney recipients from various cohorts [Table 3].

The IMPDH2 rs1 $1706052(3757 \mathrm{~T}>\mathrm{C})$ was found to be associated with increased IMPDH activity in PBMC and increased IMPDH plasma concentration, six and $12 \mathrm{~h}$, respectively, after MMF oral intake by 101 adult kidney recipients ${ }^{[78]}$. In this line of evidence, Winnicki et al. ${ }^{[79]}$ reported that $I M P D H 23757 \mathrm{~T}>\mathrm{C}$ reduced the antiproliferative effect of MPA on lymphocytes (50\% inhibition) isolated from 20 healthy volunteers. The authors suggested that this variant is associated with a poor response to MPA therapy. However, in a large cohort from the Collaborative Transplant Study, the IMPDH2 rs11706052 had no impact on MMF dose tolerated (one year) or dose achieved (three years) ${ }^{[73]}$.

IMPDH2 rs1 1706052 (3757T $>\mathrm{C})$ was also found to be associated with increased risk of AR at one year (3757C allele: OR: $3.39,95 \% \mathrm{CI}: 1.42-8.09, P=0.006$ ) in adult kidney recipients treated with MMF from the CAESAR study ${ }^{[25]}$. Conversely, the rs1 1706052 was reported to reduce the risk of AR (3757C allele: OR: 0.24, 95\%CI: 0.07-0.78, $P=0.018)$ in Brazilian adult patients ${ }^{[22]}$.

Further studies did not confirm the association of the IMPDH2 rs11706052 with AR in adult patients from different populations ${ }^{[29,71,78,80]}$, including large cohorts ${ }^{[72,73]}$, and pediatric patients ${ }^{[27]}$. Lack of association with AR was also described for IMPDH2 variants rs121434586 (Leu236Phe, c.787C>T) and rs4974081 $(-3624 \mathrm{~A}>\mathrm{G})$ in adult kidney recipients on MMF treatment from a US cohort ${ }^{[7]]}$ and the Apomygre and FDCC studies ${ }^{[72]}$.

Shah et al ${ }^{[73]}$ also reported the lack of an association of the IMPDH2 rs11706052 with long-term graft function (one year) and graft survival (five years) in a large cohort of kidney recipients on MMF therapy from the Collaborative Transplant Study.

Some studies also explored the influence of polymorphisms in $\mathrm{IMPDH} 2$ on adverse events related to MPA. Pazik et al. ${ }^{[80]}$ described an association of the IMPDH2 rs1 1706052 (3757T>C) polymorphism with increased lymphocyte counts and reduced risk of lymphopenia (3757C allele: OR: 0.32, 95\%CI: 0.11-0.90, $P=0.032$ ), but not with neutropenia in adult kidney recipients from Poland. Other studies did not confirm the influence of the rs1 1706052 variant on leukopenia in adult kidney recipients on $\mathrm{MMF}^{[71,72]}$ or EC-MPS treatment ${ }^{[29]}$. In the same way, a retrospective study reported no association of rs11706052 with leukopenia or time to leukopenia in children and young adults from the US ${ }^{[27]}$.

Other variants in $I M P D H 2$, such as rs 121434586 (Leu236Phe, c.787C $>\mathrm{T}$ ) and rs4974081 $(-3624 \mathrm{~A}>\mathrm{G}$ ), were studied, but no association with leukopenia was found in adult patients ${ }^{[71,72]}$, children, and young adults $^{[27]}$ on MMF treatment. The influence of the IMPDH2 rs11706052 on anemia was also explored but no association was found in adult patients from the Dominos Study on EC-MPS therapy ${ }^{[2]]}$.

Two studies explored MPA-related gastrointestinal adverse events and found no association of IMPDH2 rs11706052 with diarrhea in adult patients on $\mathrm{MMF}^{[80]}$ or EC-MPS treatment ${ }^{[29]}$.

Gensburger et al. ${ }^{[72]}$ found a lack of association between IMPDH2 rs11706052 and rs4974081 and the susceptibility to CMV and other infections in adult kidney recipients on MMF therapy from the Apomygre and FDCC studies. This was also reported for $I M P D H 2$ rs1 1706052 and the incidence of serious infections in adult patients on MMF treatment from Poland ${ }^{[80]}$. Pazik et al. ${ }^{[75]}$ also studied how the presence of IMPDH2 rs11706052 could change the BMI of adult kidney recipients on MMF treatment in a timedependent manner, but no association was found with BMI change over five years post-transplantation. 


\section{CLINICAL IMPLEMENTATION}

Increasing knowledge in pharmacogenomics and its clinical implementations depend on several factors, including the robustness of the studies, sample size, and reproducibility of the results in different populations. The Pharmacogenomics Knowledge Base (PharmGKB; www.pharmgkb.org) aims to collect, encode and disseminate understanding of human genetic variations on drug responses ${ }^{[81,82]}$, and most of the existing pharmacogenetic information is compiled in this database ${ }^{[83]}$. For clinical annotations, PharmGKB curators determine "levels of evidence" score that is a measure of confidence in the variantdrug associations using well-defined criteria based on careful literature review. This score has four levels, from 1 to $4(1 \mathrm{~A}, 1 \mathrm{~B}, 2 \mathrm{~A}, 2 \mathrm{~B}, 3$ and 4$), 1 \mathrm{~A}$ being the highest scientific evidence ${ }^{[81,82]}$.

In kidney transplantation, the clinical annotations registered in PharmGKB include the following variants implicated in MPA efficacy, metabolism or toxicity: IMPDH1 $\mathrm{rs}_{22} 28293^{[13,71-74]}$ and $\mathrm{rs} 2278294^{[13,71-74]}$, IMPDH2 rs1 $1706052^{[25,72,73,78-80]}, A B C B 1 \mathrm{rs} 2032582^{[25]}, A B C C 2 \mathrm{rs}_{2} 273697^{[64,83]}, \mathrm{rs}_{3740066^{[83]}}$, and $\mathrm{rs} 717620^{[64]^{\prime}}$,

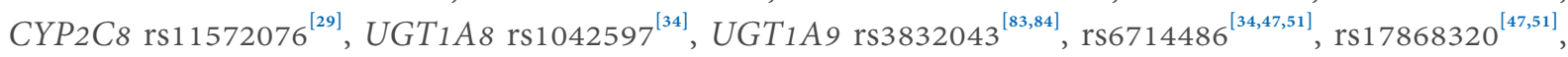

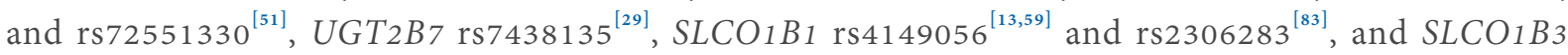
rs7311358 ${ }^{[14,65]}$ and $\operatorname{rs} 4149117^{[13,14,59,65]}$. All these variants are assigned as evidence level 3 with exception of SLCO1B3 rs4149117, which is level 4. Level 3 means "a single significant (not yet replicated) study or annotation for a variant-drug combination evaluated in multiple studies but lacking clear evidence of an association", whereas level 4 is based on "a case report; on a study that did not achieve significance but is biologically plausible; or on in vitro, molecular, or functional assay evidence" ${ }^{\text {[81,82] }}$.

Although the data reviewed here highlight the importance of pharmacogenomics in the variability of the response to MPA, these clinical associations are not strong enough to be used for clinical translation ${ }^{[85]}$ and more evidence is needed to clarify the level of contribution of pharmacogenomics in kidney transplant patients treated with MPA.

Moreover, it is important to mention that three regulatory agencies, Food and Drug Administration (FDA, US), Pharmaceuticals and Medical Devices Agency (PMDA, Japan) and Health Canada (Santé, Canada) (HCSC), recommend drug labeling for MPA as an "actionable PGx" (genetic testing not required) for patients with rare hereditary deficiency of hypoxanthine-guanine phosphoribosyl-transferase, such as Lesch-Nyhan and Kelley-Seegmiller syndrome, because it may cause an exacerbation of disease symptoms characterized by the overproduction and accumulation of uric $\operatorname{acid}^{[81,82,86]}$.

\section{FINAL CONSIDERATIONS}

The genotype-phenotype associations reviewed here showed that genetic influence on MPA treatment seems to be small, especially due to inconsistency between studies. However, many factors need to be considered.

The strategies applied to MPA dosing were different between studies, which included fixed dosing ("onedose-fits-all") and dosing to a therapeutic range (therapeutic drug monitoring-TDM). MPA AUC ${ }_{0-12}$ has been recommended as the best marker for dose adjustment ${ }^{[87]}$. However, unlike other drugs such as tacrolimus, MPA trough level showed a poor correlation with AUC ${ }^{[87-89]}$. TDM use for MPA is drawing much attention, but it is still controversial ${ }^{[3]}$. It is known that TDM can contribute to correct the variability in MPA exposure ${ }^{[89,90]}$. Therefore, TDM of MPA can minimize the genetic influence on the efficacy and safety of therapy.

Moreover, the studies included in this review are heterogeneous in some aspects, such as population (sample size, ethnics, age, etc.), inherent characteristics of the clinical approach, immunosuppressive scheme and 
follow-up time, time after transplantation, definition of the analyzed events (for example, AR, DGF or adverse events), and analytical methods for measurement of the MPA exposure.

Together, these heterogeneities demonstrate the limitations of this review for pointing out the pharmacogenetic biomarkers useful for clinical applications of MPA in kidney transplantation.

\section{CONCLUSION}

Individualized treatment can contribute to improve efficacy and decrease the toxicity of immunosuppressive drugs. Here, the influence of genetic variants on MPA pharmacokinetics and pharmacodynamics in kidney transplant recipients was reviewed. The combination of multiple drugs, the different sample sizes, and the lack of association consistency between studies have been important challenges of MPA pharmacogenomics. Together they limit the conclusions and clinical applications of MPA pharmacogenomics in kidney transplantation. Currently, further pharmacogenomic studies are needed to elucidate the contribution of genetic background to the effectiveness and safety of MPA therapy.

\section{DECLARATIONS}

\section{Acknowledgments}

Genvigir FDV and Hirata TDC were recipients of a fellowship from FAPESP, Brazil. Hirata MH and Hirata $\mathrm{RDC}$ are recipients of a fellowship from CNPq, Brazil.

\section{Authors' contributions}

Made substantial contributions to the conception and design of the study and performed data analysis and interpretation: Genvigir FDV, Cerda A, Hirata RDC

Prepared the figure and critically reviewed the manuscript: Hirata TDC, Hirata MH

\section{Availability of data and materials}

Not applicable.

\section{Financial support and sponsorship}

None.

\section{Conflicts of interest}

All authors declared that there are no conflicts of interest.

\section{Ethical approval and consent to participate}

Not applicable.

\section{Consent for publication}

Not applicable.

\section{Copyright}

(c) The Author(s) 2020.

\section{REFERENCES}

1. Bentata Y. Mycophenolates: the latest modern and potent immunosuppressive drugs in adult kidney transplantation: what we should know about them? Artif Organs 2020;44:561-76.

2. Guo M, Wang ZJ, Yang HW, Meng L, Tan RY, et al. Influence of genetic polymorphisms on mycophenolic acid pharmacokinetics and patient outcomes in renal transplantation. Curr Drug Metab 2018;19:1199-205.

3. Staatz CE, Tett SE. Pharmacology and toxicology of mycophenolate in organ transplant recipients: an update. Arch Toxicol 2014;88:1351-89. 
4. da Silva MB, da Cunha FF, Terra FF, Camara NO. Old game, new players: Linking classical theories to new trends in transplant immunology. World J Transplant 2017;7:1-25.

5. Cooper M, Salvadori M, Budde K, Oppenheimer F, Sollinger H, et al. Enteric-coated mycophenolate sodium immunosuppression in renal transplant patients: efficacy and dosing. Transplant Rev (Orlando) 2012;26:233-40.

6. Fujiyama N, Miura M, Kato S, Sone T, Isobe M, et al. Involvement of carboxylesterase 1 and 2 in the hydrolysis of mycophenolate mofetil. Drug Metab Dispos 2010;38:2210-7.

7. Burg M, Säemann MD, Wieser C, Kramer S, Fischer W, et al. Enteric-coated mycophenolate sodium reduces gastrointestinal symptoms in renal transplant patients. Transplant Proc 2009;41:4159-64.

8. van Gelder T, Hesselink DA. Mycophenolate revisited. Transpl Int 2015;28:508-15.

9. Bernard O, Guillemette C. The main role of UGT1A9 in the hepatic metabolism of mycophenolic acid and the effects of naturally occurring variants. Drug Metab Dispos 2004;32:775-8.

10. Meier-Kriesche HU, Shaw LM, Korecka M, Kaplan B. Pharmacokinetics of mycophenolic acid in renal insufficiency. Ther Drug Monit 2000;22:27-30.

11. Shipkova M, Armstrong VW, Oellerich M, Wieland E. Acyl glucuronide drug metabolites: toxicological and analytical implications. Ther Drug Monit 2003;25:1-16.

12. Picard N, Cresteil T, Prémaud A, Marquet P. Characterization of a phase 1 metabolite of mycophenolic acid produced by CYP3A4/5. Ther Drug Monit 2004;26:600-8.

13. Michelon H, König J, Durrbach A, Quteineh L, Verstuyft C, et al. SLCO1B1 genetic polymorphism influences mycophenolic acid tolerance in renal transplant recipients. Pharmacogenomics 2010;11:1703-13.

14. Picard N, Yee SW, Woillard JB, Lebranchu Y, Le Meur Y, et al. The role of organic anion-transporting polypeptides and their common genetic variants in mycophenolic acid pharmacokinetics. Clin Pharmacol Ther 2010;87:100-8.

15. Westley IS, Brogan LR, Morris RG, Evans AM, Sallustio BC. Role of Mrp2 in the hepatic disposition of mycophenolic acid and its glucuronide metabolites: effect of cyclosporine. Drug Metab Dispos 2006;34:261-6.

16. Kobayashi M, Saitoh H, Kobayashi M, Tadano K, Takahashi Y, et al. Cyclosporin A, but not tacrolimus, inhibits the biliary excretion of mycophenolic acid glucuronide possibly mediated by multidrug resistance-associated protein 2 in rats. J Pharmacol Exp Ther 2004;309:1029-35.

17. Jetter A, Kullak-Ublick GA. Drugs and hepatic transporters: a review. Pharmacol Res 2020;154:104234.

18. Wang J, Figurski M, Shaw LM, Burckart GJ. The impact of P-glycoprotein and Mrp2 on mycophenolic acid levels in mice. Transpl Immunol 2008;19:192-6.

19. Patel CG, Ogasawara K, Akhlaghi F. Mycophenolic acid glucuronide is transported by multidrug resistance-associated protein 2 and this transport is not inhibited by cyclosporine, tacrolimus or sirolimus. Xenobiotica 2013;43:229-35.

20. Wang D, Zou L, Jin Q, Hou J, Ge G, et al. Human carboxylesterases: a comprehensive review. Acta Pharm Sin B 2018;8:699-712.

21. Fujiyama N, Miura M, Satoh S, Inoue K, Kagaya H, et al. Influence of carboxylesterase 2 genetic polymorphisms on mycophenolic acid pharmacokinetics in Japanese renal transplant recipients. Xenobiotica 2009;39:407-14.

22. Cilião HL, Camargo-Godoy RBO, Souza MF, Zanuto A, Delfino VDA, et al. Polymorphisms in IMPDH2, UGT2B7, and CES2 genes influence the risk of graft rejection in kidney transplant recipients taking mycophenolate mofetil. Mutat Res Genet Toxicol Environ Mutagen 2018;836:97-102.

23. Satoh S, Tada H, Murakami M, Tsuchiya N, Li Z, et al. Circadian pharmacokinetics of mycophenolic Acid and implication of genetic polymorphisms for early clinical events in renal transplant recipients. Transplantation 2006;82:486-93.

24. Genvigir FDV, Campos-Salazar AB, Felipe CR, Tedesco-Silva H Jr, Medina-Pestana JO, et al. CYP3A5*3 and CYP2C $8 * 3$ variants influence exposure and clinical outcomes of tacrolimus-based therapy. Pharmacogenomics 2020;21:7-21.

25. Grinyó J, Vanrenterghem Y, Nashan B, Vincenti F, Ekberg H, et al. Association of four DNA polymorphisms with acute rejection after kidney transplantation. Transpl Int 2008;21:879-91.

26. Grenda R, Prokurat S, Ciechanowicz A, Piatosa B, Kaliciński P. Evaluation of the genetic background of standard-immunosuppressantrelated toxicity in a cohort of 200 paediatric renal allograft recipients - a retrospective study. Ann Transplant 2009;14:18-24.

27. Varnell CD, Fukuda T, Kirby CL, Martin LJ, Warshaw BL, et al. Mycophenolate mofetil-related leukopenia in children and young adults following kidney transplantation: influence of genes and drugs. Pediatr Transplant 2017;21:e13033.

28. Jacobson PA, Schladt D, Oetting WS, Leduc R, Guan W, et al; DeKAF investigators. Genetic determinants of mycophenolate-related anemia and leukopenia after transplantation. Transplantation 2011;91:309-16.

29. Woillard JB, Picard N, Thierry A, Touchard G, Marquet P; DOMINOS study group. Associations between polymorphisms in target, metabolism, or transport proteins of mycophenolate sodium and therapeutic or adverse effects in kidney transplant patients. Pharmacogenet Genomics 2014;24:256-62.

30. Genvigir FDV, Nishikawa AM, Felipe CR, Tedesco-Silva H Jr, Oliveira N, et al. Influence of ABCC2, CYP2C8, and CYP2J2 polymorphisms on tacrolimus and mycophenolate sodium-based treatment in brazilian kidney transplant recipients. Pharmacotherapy 2017;37:535-45.

31. Bernard O, Tojcic J, Journault K, Perusse L, Guillemette C. Influence of nonsynonymous polymorphisms of UGT1A8 and UGT2B7 metabolizing enzymes on the formation of phenolic and acyl glucuronides of mycophenolic acid. Drug Metab Dispos 2006;34:1539-45.

32. Meech R, Hu DG, McKinnon RA, Mubarokah SN, Haines AZ, et al. The UDP-glycosyltransferase (UGT) superfamily: new members, new functions, and novel paradigms. Physiol Rev 2019;99:1153-222.

33. Inoue K, Miura M, Satoh S, Kagaya H, Saito M, et al. Influence of UGT1A7 and UGT1A9 intronic I399 genetic polymorphisms on 
mycophenolic acid pharmacokinetics in Japanese renal transplant recipients. Ther Drug Monit 2007;29:299-304.

34. Woillard JB, Rerolle JP, Picard N, Rousseau A, Drouet M, et al. Risk of diarrhoea in a long-term cohort of renal transplant patients given mycophenolate mofetil: the significant role of the UGT1A8 2 variant allele. Br J Clin Pharmacol 2010;69:675-83.

35. Xie XC, Li J, Wang HY, Li HL, Liu J, et al. Associations of UDP-glucuronosyltransferases polymorphisms with mycophenolate mofetil pharmacokinetics in Chinese renal transplant patients. Acta Pharmacol Sin 2015;36:644-50.

36. Kagaya H, Inoue K, Miura M, Satoh S, Saito M, et al. Influence of UGT1A8 and UGT2B7 genetic polymorphisms on mycophenolic acid pharmacokinetics in Japanese renal transplant recipients. Eur J Clin Pharmacol 2007;63:279-88.

37. Geng F, Jiao Z, Dao YJ, Qiu XY, Ding JJ, et al. The association of the UGT1A8, SLCO1B3 and ABCC2/ABCG2 genetic polymorphisms with the pharmacokinetics of mycophenolic acid and its phenolic glucuronide metabolite in Chinese individuals. Clin Chim Acta 2012;413:683-90.

38. Li LQ, Chen DN, Li CJ, Li QP, Chen Y, et al. Impact of UGT2B7 and ABCC2 genetic polymorphisms on mycophenolic acid metabolism in Chinese renal transplant recipients. Pharmacogenomics 2018;19:1323-34.

39. van Schaik RH, van Agteren M, de Fijter JW, Hartmann A, Schmidt J, et al. UGT1A9 -275T >A/-2152C >T polymorphisms correlate with low MPA exposure and acute rejection in MMF/tacrolimus-treated kidney transplant patients. Clin Pharmacol Ther 2009;86:319-27.

40. Zhao W, Fakhoury M, Deschênes G, Roussey G, Brochard K, et al. Population pharmacokinetics and pharmacogenetics of mycophenolic acid following administration of mycophenolate mofetil in de novo pediatric renal-transplant patients. J Clin Pharmacol 2010;50:1280-91.

41. Vu D, Tellez-Corrales E, Yang J, Qazi Y, Shah T, et al. Genetic polymorphisms of UGT1A8, UGT1A9 and HNF-1 $\alpha$ and gastrointestinal symptoms in renal transplant recipients taking mycophenolic acid. Transpl Immunol 2013;29:155-61.

42. Betônico GN, Abbud-Filho M, Goloni-Bertollo EM, Alvarenga MP, Guillemette C, et al. Influence of UDP-glucuronosyltransferase polymorphisms on mycophenolate mofetil-induced side effects in kidney transplant patients. Transplant Proc 2008;40:708-10.

43. Prausa SE, Fukuda T, Maseck D, Curtsinger KL, Liu C, et al. UGT genotype may contribute to adverse events following medication with mycophenolate mofetil in pediatric kidney transplant recipients. Clin Pharmacol Ther 2009;85:495-500.

44. Girard H, Court MH, Bernard O, Fortier LC, Villeneuve L, et al. Identification of common polymorphisms in the promoter of the UGT1A9 gene: evidence that UGT1A9 protein and activity levels are strongly genetically controlled in the liver. Pharmacogenetics 2004;14:501-15.

45. Kuypers DR, de Jonge H, Naesens M, de Loor H, Halewijck E, et al. Current target ranges of mycophenolic acid exposure and drugrelated adverse events: a 5-year, open-label, prospective, clinical follow-up study in renal allograft recipients. Clin Ther 2008;30:673-83.

46. Sánchez-Fructuoso AI, Maestro ML, Calvo N, Viudarreta M, Pérez-Flores I, et al. The prevalence of uridine diphosphateglucuronosyltransferase 1A9 (UGT1A9) gene promoter region single-nucleotide polymorphisms T-275A and C-2152T and its influence on mycophenolic acid pharmacokinetics in stable renal transplant patients. Transplant Proc 2009;41:2313-6.

47. Kuypers DR, Naesens M, Vermeire S, Vanrenterghem Y. The impact of uridine diphosphate-glucuronosyltransferase 1A9 (UGT1A9) gene promoter region single-nucleotide polymorphisms T-275A and C-2152T on early mycophenolic acid dose-interval exposure in de novo renal allograft recipients. Clin Pharmacol Ther 2005;78:351-61.

48. Baldelli S, Merlini S, Perico N, Nicastri A, Cortinovis M, et al. C-440T/T-331C polymorphisms in the UGT1A9 gene affect the pharmacokinetics of mycophenolic acid in kidney transplantation. Pharmacogenomics 2007;8:1127-41.

49. Kiang TKL, Partovi N, Shapiro RJ, Berman JM, Collier AC, et al. Regression and genomic analyses on the association between dosenormalized mycophenolic acid exposure and absolute neutrophil count in steroid-free, de novo kidney transplant recipients. Clin Drug Investig 2018;38:1011-22.

50. Yu ZC, Zhou PJ, Wang XH, Françoise B, Xu D, et al. Population pharmacokinetics and Bayesian estimation of mycophenolic acid concentrations in Chinese adult renal transplant recipients. Acta Pharmacol Sin 2017;38:1566-79.

51. Pazik J, Ołdak M, Dąbrowski M, Lewandowski Z, Sitarek E, et al. Association of UDP-glucuronosyltransferase 1A9 (UGT1A9) gene polymorphism with kidney allograft function. Ann Transplant 2011;16:69-73.

52. Pazik J, Ołdak M, Lewandowski Z, Dąbrowski M, Podgórska M, et al. Recipient uridine 5'-diphospho-glucuronosyltransferase UGT1A9 c. $98 \mathrm{~T}>\mathrm{C}$ variant determines transplanted kidney filtration rate. Transplant Proc 2014;46:2678-82.

53. Djebli N, Picard N, Rérolle JP, Le Meur Y, Marquet P. Influence of the UGT2B7 promoter region and exon 2 polymorphisms and comedications on Acyl-MPAG production in vitro and in adult renal transplant patients. Pharmacogenet Genomics 2007;17:321-30.

54. van Agteren M, Armstrong VW, van Schaik RH, de Fijter H, Hartmann A, et al. AcylMPAG plasma concentrations and mycophenolic acid-related side effects in patients undergoing renal transplantation are not related to the UGT2B7-840G $>$ A gene polymorphism. Ther Drug Monit 2008;30:439-44.

55. Cattaneo D, Perico N, Gaspari F, Gotti E, Remuzzi G. Glucocorticoids interfere with mycophenolate mofetil bioavailability in kidney transplantation. Kidney Int 2002;62:1060-7.

56. Duguay Y, Báár C, Skorpen F, Guillemette C. A novel functional polymorphism in the uridine diphosphate-glucuronosyltransferase 2B7 promoter with significant impact on promoter activity. Clin Pharmacol Ther 2004;75:223-33.

57. Yoo EC, Alvarez-Elías AC, Todorova EK, Filler G. Developmental changes of MPA exposure in children. Pediatr Nephrol 2016;31:975-82.

58. Bruhn O, Cascorbi I. Polymorphisms of the drug transporters ABCB1, ABCG2, ABCC2 and ABCC3 and their impact on drug bioavailability and clinical relevance. Expert Opin Drug Metab Toxicol 2014;10:1337-54.

59. Bouamar R, Hesselink DA, van Schaik RH, Weimar W, van der Heiden IP, et al. Mycophenolic acid-related diarrhea is not associated with polymorphisms in SLCO1B nor with ABCB1 in renal transplant recipients. Pharmacogenet Genomics 2012;22:399-407.

60. Treiber A, Schneiter R, Häusler S, Stieger B. Bosentan is a substrate of human OATP1B1 and OATP1B3: inhibition of hepatic uptake as the common mechanism of its interactions with cyclosporin A, rifampicin, and sildenafil. Drug Metab Dispos 2007;35:1400-7. 
61. Staatz CE, Goodman LK, Tett SE. Effect of CYP3A and ABCB1 single nucleotide polymorphisms on the pharmacokinetics and pharmacodynamics of calcineurin inhibitors: Part I. Clin Pharmacokinet 2010;49:141-75.

62. Naesens M, Kuypers DR, Verbeke K, Vanrenterghem Y. Multidrug resistance protein 2 genetic polymorphisms influence mycophenolic acid exposure in renal allograft recipients. Transplantation 2006;82:1074-84.

63. Lloberas N, Torras J, Cruzado JM, Andreu F, Oppenheimer F, et al; Spanish Pharmacogenetic Symphony Substudy Group. Influence of MRP2 on MPA pharmacokinetics in renal transplant recipients-results of the pharmacogenomic substudy within the symphony study. Nephrol Dial Transplant 2011;26:3784-93.

64. Božina N, Lalić Z, Nađ-Škegro S, Borić-Bilušić A, Božina T, et al. Steady-state pharmacokinetics of mycophenolic acid in renal transplant patients: exploratory analysis of the effects of cyclosporine, recipients' and donors' ABCC2 gene variants, and their interactions. Eur J Clin Pharmacol 2017;73:1129-40.

65. Miura M, Satoh S, Inoue K, Kagaya H, Saito M, et al. Influence of SLCO1B1, 1B3, 2B1 and ABCC2 genetic polymorphisms on mycophenolic acid pharmacokinetics in Japanese renal transplant recipients. Eur J Clin Pharmacol 2007;63:1161-9.

66. Colom H, Lloberas N, Andreu F, Caldés A, Torras J, et al. Pharmacokinetic modeling of enterohepatic circulation of mycophenolic acid in renal transplant recipients. Kidney Int 2014;85:1434-43.

67. Li TT, An JX, Xu JY, Tuo BG. Overview of organic anion transporters and organic anion transporter polypeptides and their roles in the liver. World J Clin Cases 2019;7:3915-33.

68. Song W, Li D, Tao L, Luo Q, Chen L. Solute carrier transporters: the metabolic gatekeepers of immune cells. Acta Pharm Sin B 2020;10:61-78.

69. Kalliokoski A, Niemi M. Impact of OATP transporters on pharmacokinetics. Br J Pharmacol 2009;158:693-705.

70. Wu TY, Peng Y, Pelleymounter LL, Moon I, Eckloff BW, et al. Pharmacogenetics of the mycophenolic acid targets inosine monophosphate dehydrogenases IMPDH1 and IMPDH2: gene sequence variation and functional genomics. Br J Pharmacol 2010;161:1584-98.

71. Wang J, Yang JW, Zeevi A, Webber SA, Girnita DM, et al. IMPDH1 gene polymorphisms and association with acute rejection in renal transplant patients. Clin Pharmacol Ther 2008;83:711-7.

72. Gensburger O, Van Schaik RH, Picard N, Le Meur Y, Rousseau A, et al. Polymorphisms in type I and II inosine monophosphate dehydrogenase genes and association with clinical outcome in patients on mycophenolate mofetil. Pharmacogenet Genomics 2010;20:537-43.

73. Shah S, Harwood SM, Döhler B, Opelz G, Yaqoob MM. Inosine monophosphate dehydrogenase polymorphisms and renal allograft outcome. Transplantation 2012;94:486-91.

74. Kagaya H, Miura M, Saito M, Habuchi T, Satoh S. Correlation of IMPDH1 gene polymorphisms with subclinical acute rejection and mycophenolic acid exposure parameters on day 28 after renal transplantation. Basic Clin Pharmacol Toxicol 2010;107:631-6.

75. Pazik J, Lewandowski Z, Nowacka Cieciura E, Ołdak M, Podgórska M, et al. Malnutrition risk in kidney recipients treated with mycophenolate mofetil is associated with IMPDH1 rs2278294 polymorphism. Transplant Proc 2018;50:1794-7.

76. Wang J, Zeevi A, Webber S, Girnita DM, Addonizio L, et al. A novel variant L263F in human inosine 5'-monophosphate dehydrogenase 2 is associated with diminished enzyme activity. Pharmacogenet Genomics 2007;17:283-90.

77. Mohamed MF, Frye RF, Langaee TY. Interpopulation variation frequency of human inosine 5'-monophosphate dehydrogenase type II (IMPDH2) genetic polymorphisms. Genet Test 2008;12:513-6.

78. Sombogaard F, van Schaik RH, Mathot RA, Budde K, van der Werf M, et al. Interpatient variability in IMPDH activity in MMF-treated renal transplant patients is correlated with IMPDH type II 3757T>C polymorphism. Pharmacogenet Genomics 2009;19:626-34.

79. Winnicki W, Weigel G, Sunder-Plassmann G, Bajari T, Winter B, et al. An inosine 5'-monophosphate dehydrogenase 2 single-nucleotide polymorphism impairs the effect of mycophenolic acid. Pharmacogenomics J 2010;10:70-6.

80. Pazik J, Ołdak M, Podgórska M, Lewandowski Z, Sitarek E, et al. Lymphocyte counts in kidney allograft recipients are associated with IMPDH2 3757T $>$ C gene polymorphism. Transplant Proc 2011;43:2943-5.

81. McDonagh EM, Whirl-Carrillo M, Garten Y, Altman RB, Klein TE. From pharmacogenomic knowledge acquisition to clinical applications: the PharmGKB as a clinical pharmacogenomic biomarker resource. Biomark Med 2011;5:795-806.

82. Whirl-Carrillo M, McDonagh EM, Hebert JM, Gong L, Sangkuhl K, et al. Pharmacogenomics knowledge for personalized medicine. Clin Pharmacol Ther 2012;92:414-7.

83. Han N, Yun HY, Kim IW, Oh YJ, Kim YS, et al. Population pharmacogenetic pharmacokinetic modeling for flip-flop phenomenon of enteric-coated mycophenolate sodium in kidney transplant recipients. Eur J Clin Pharmacol 2014;70:1211-9.

84. Zhang WX, Chen B, Jin Z, Yu Z, Wang X, et al. Influence of uridine diphosphate (UDP)-glucuronosyltransferases and ABCC2 genetic polymorphisms on the pharmacokinetics of mycophenolic acid and its metabolites in Chinese renal transplant recipients. Xenobiotica 2008;38:1422-36.

85. Olivera G, Sendra L, Herrero MJ, Berlanga P, Gargallo P, et al. Pharmacogenetics implementation in the clinics: information and guidelines for germline variants. CDR 2019

86. Messina E, Micheli V, Giacomello A. Guanine nucleotide depletion induces differentiation and aberrant neurite outgrowth in human dopaminergic neuroblastoma lines: a model for basal ganglia dysfunction in Lesch-Nyhan disease. Neurosci Lett 2005;375:97-100.

87. Zhang J, Sun Z, Zhu Z, Yang J, Kang J, et al. Pharmacokinetics of mycophenolate mofetil and development of limited sampling strategy in early kidney transplant recipients. Front Pharmacol 2018;9:908.

88. Marquet P. Counterpoint: is pharmacokinetic or pharmacodynamic monitoring of calcineurin inhibition therapy necessary? Clin Chem 2010;56:736-9. 
89. Metz DK, Holford N, Kausman JY, Walker A, Cranswick N, et al. Optimizing mycophenolic acid exposure in kidney transplant recipients: time for target concentration intervention. Transplantation 2019;103:2012-30.

90. Picard N, Bergan S, Marquet P, van Gelder T, Wallemacq P, et al. Pharmacogenetic biomarkers predictive of the pharmacokinetics and pharmacodynamics of immunosuppressive drugs. Ther Drug Monit 2016;38 Suppl 1:S57-69. 